\title{
Joint Optimal Sensor Selection and Scheduling in Dynamic Spectrum Access Networks
}

\author{
Alexander W. Min, Member, IEEE, and Kang G. Shin, Fellow, IEEE
}

\begin{abstract}
Spectrum sensing is key to the realization of dynamic spectrum access. To protect primary users' communications from the interference caused by secondary users, spectrum sensing must meet the strict detectability requirements set by regulatory bodies, such as the FCC. Such strict detection requirements, however, can hardly be achieved using PHY-layer sensing techniques alone with one-time sensing by only a single sensor. In this paper, we jointly exploit two MAC-layer sensing methods-cooperative sensing and sensing scheduling - to improve spectrum sensing performance, while incurring minimum sensing overhead. While these sensing methods have been studied individually, little has been done on their combinations and the resulting benefits. Specifically, we propose to construct a profile of the primary signal's RSSs and design a simple, yet near-optimal, incumbent detection rule. Based on this constructed RSS profile, we develop an algorithm to find 1) an optimal set of sensors; 2) an optimal point at which to stop scheduling additional sensing; and 3) an optimal sensing duration for one-time sensing, so as to make a tradeoff between detection performance and sensing overhead. Our evaluation results show that the proposed sensing algorithms reduce the sensing overhead by up to 65 percent, while meeting the requirements of both false-alarm and misdetection probabilities of less than 0.01 .
\end{abstract}

Index Terms-Cognitive radio, cooperative sensing, dynamic spectrum access, sensor selection, sensing scheduling

\section{INTRODUCTION}

$\mathrm{D}$ YNAMIC spectrum access (DSA) is a promising technology that can help alleviate the impending spectrumscarcity problem, thus promoting the introduction of new wireless services and meeting growing user demands. In DSA networks, (license-exempt) secondary users (SUs) identify temporarily unused licensed spectrum bands, and opportunistically access them as long as SUs do not interfere with legacy primary users' (PUs') communications. Recent measurement studies [1], [2] have indicated that a significant portion of licensed spectrum bands are highly underutilized, thus making DSA an attractive means to greatly improve spectrum efficiency. To realize such potential benefits of DSA, it is of the utmost importance to efficiently and reliably detect spectrum opportunities or white spaces.

In DSA networks, there are various ways to discover spectrum opportunities, such as spectrum sensing [3], [4], beacon protocol [5], [6], or geolocation database [7], [8], [9]. Spectrum sensors detect the presence or absence of a primary signal in a target spectrum band. To protect PUs' communications, spectrum sensing must meet strict requirements set by regulatory bodies, such as the Federal Communications Commission (FCC). For example, in the IEEE 802.22 Wireless Regional Area Networks (WRANs) [10], [11], a primary signal as weak as $-20 \mathrm{~dB}$ must be

- A.W. Min is with the Systems Architecture Lab, Intel Labs, 2111 N.E. 25th Avenue, Hillsboro, OR 97124.E-mail: alexander.w.min@intel.com.

- K.G. Shin is with the Real-Time Computing Laboratory (RTCL), Department of Electrical Engineering and Computer Science, The University of Michigan, 2260 Hayward Street, Ann Arbor, MI 481092121. E-mail:kgshin@eecs.umich.edu.

Manuscript received 9 Sept. 2011; revised 30 Mar. 2012; accepted 27 Apr. 2012; published online 8 May 2012.

For information on obtaining reprints of this article, please send e-mail to: tmc@computer.org, and reference IEEECS Log Number TMC-2011-09-0493. Digital Object Identifier no. 10.1109/TMC.2012.116. detected with high accuracy, i.e., both false-alarm and misdetection probabilities must be less than 10 percent [12]. Unfortunately, this stringent requirement cannot be met using one-time sensing ${ }^{1}$ with a single sensor, regardless of the underlying PHY-layer sensing schemes, e.g., energy/ feature detection [13], [14], [15]. A second method for detecting a primary signal, called the beacon protocol, has also been proposed to detect the presence of a primary signal more efficiently. However, the beacon protocol requires legacy devices to be equipped with an external beacon device, which may not be feasible for already widely deployed legacy systems because of the high cost involved. Moreover, such modifications violate the basic premise of DSA-opportunistic spectrum access should require no modification to legacy systems. Thus, the beacon protocol cannot obviate the need for spectrum sensing. Alternatively, a geolocation database can be used to identify spectrum availability at a given time and location [16]. Such a database can be constructed and maintained by regulatory bodies, such as the FCC in the US or Ofcom in the United Kingdom, or by a trusted third party. A database can be used for spectrum bands on which PU activities are predictable, e.g., DTV signals with long ON/OFF periods. Moreover, it can reduce the cost of large-scale sensor deployment. In fact, the FCC recently mandated the use of a geolocation database for accessing TV band white spaces [17]. Algorithms and frameworks for implementing geolocation databases for TV spectrum bands are currently under development [7], [9]. However, construction of a database with a complete spectrum map either via measurement or propagation modeling, may require a considerable amount of time. Examining the database to check spectrum availability also consumes system resources. Moreover,

1. We use the term "one-time sensing" to mean sensing only once. 
spectrum sensing may still lead to better spectrum utilization compared to the use of a geolocation database.

In this paper, we focus on improving spectrum-sensing performance via joint design of two MAC-layer sensing methods, namely cooperative sensing and sensing scheduling. This joint design allows SUs not only to overcome the performance deficiency of PHY-layer sensing, but also to make the tradeoff between performance gain and sensing overhead. In cooperative sensing, a fusion center (base station) directs multiple sensors at different locations to perform spectrum sensing simultaneously during each sensing (quiet) period, thus exploiting sensor location diversity [3], [18], [19], [20], [21], [22], [23], [24]. Scheduling sensing also aims to improve detection performance by having SUs perform spectrum sensing at various time intervals, thereby exploiting temporal variations in received signal strengths (RSSs) at each sensor [4], [25]. However, all SUs (sensors) ${ }^{2}$ must remain silent during sensing periods so that SU signals are not misinterpreted as primary signals [26]. These periods of silence waste precious resources, such as energy and time, and ultimately degrade the quality-of-service (QoS) of SU communications. Therefore, the fusion center must carefully select a set of cooperative sensors and optimally schedule sensing periods so as to minimize sensing-induced interruptions, while guaranteeing the required detection performance, even for weak primary signals.

To address this practical challenge, we propose an efficient spectrum-sensing framework that exploits both spatial and temporal variations in sensing reports (i.e., received primary signal strengths) to minimize the sensing overhead subject to the detectability requirements. In particular, we focus on large-scale PU detection in infrastructure-based secondary networks (e.g., IEEE 802.22 WRANs) and address the following three key issues in MAC-layer spectrum sensing: 1) which sensors to use for cooperative sensing; 2) how to incorporate their heterogeneous sensitivities in data fusion; and 3) how to minimally schedule spectrum sensing. To answer these questions, we first introduce a new concept of spatial RSS profiling for the incumbent signal on a given spectrum band. Because the fusion center collects sensing results from sensors at different locations, it can construct a unique RSS profile for an incumbent signal. We show that such RSS profiles can be approximated as multivariate Gaussian when the primary signal is very weak. This Gaussian approximation allows the fusion center to develop a simple, yet near-optimal linear data-fusion rule via a linear discriminant analysis (LDA). We then characterize the network condition under which scheduling the sensing multiple times is required. We also demonstrate that there is no correlation in the sensing results even among neighboring sensors in stationary DSA networks using a software-defined radio testbed. Finally, we present algorithms that minimize sensing overhead via: 1) an optimal selection of sensors for cooperative sensing; 2) optimal sensing duration; and 3) the optimal time to stop scheduling additional sensing periods.

2. We use the terms SUs and sensors interchangeably since we focus on the sensing functionality of SUs in this paper.
The remainder of this paper is organized as follows: Section 2 reviews related work on spectrum sensing in DSA networks. Section 3 introduces a DSA network model and the energy-detection technique, followed by our approach to exploiting spatiotemporal variations in sensing reports for spectrum sensing. Section 4 presents our RSS-profilebased detection scheme for one-time sensing and its theoretical performance. Section 5 first shows that there is no temporal correlation in sensing reports among stationary sensors, and then it introduces our cooperative sensing algorithms designed to select an optimal set of sensors and to find an optimal time to stop sensing. Section 6 evaluates the performance of the proposed algorithms, and Section 7 concludes the paper.

\section{Related Work}

Various aspects of cooperative sensing have been studied, such as cooperation gain [3], [19], sensor selection [27], security [28], [29], [30], and performance-overhead tradeoffs [31], [32], [33]. The benefits of sensor collaboration have been reported to diminish as the degree of shadowing correlation among sensors increases [3], [19], [21], [34]. To minimize this detrimental effect of shadowing correlation on cooperative sensing, several sensor-selection algorithms have been introduced. For example, Selén et al. [27] proposed heuristic algorithms for selecting an uncorrelated set of sensors, given different levels of information about sensor locations. In a similar vein, Kim and Shin [4] suggested selecting sensors based on their geographical separation, so as to make the sensors uncorrelated with each other. While these approaches seek to avoid shadow fading correlation among sensors, we show that when sensors are stationary, there is virtually no temporal correlation in sensing reports (see Section 5). An extensive survey of cooperative sensing in DSA networks can be found in [35].

Sensing scheduling has also been studied as an efficient way of improving incumbent detection performance [36], [37], [38], [39], [40]. For example, Hoang and Liang [36] developed an adaptive sensing scheduling mechanism that takes into account both time-varying channel and traffic conditions. In the IEEE 802.22 WRAN standard draft, a two-stage sensing mechanism has been proposed to provide flexible scheduling of quiet periods [41]. Recently, a sequential hypothesis testing framework has been proposed as an attractive way to minimize the sensing delay for given detection requirements (in terms of falsealarm and misdetection probabilities). Lai et al. [37] presented sequential detection of primary signals using the cumulative sum (CUSUM) algorithm. Similar to our work, Zou et al. [40] proposed a sensing scheduling scheme based on the framework of sequential probability ratio testing (SPRT) under the assumption of unknown primary signal characteristics. However, the interactions between cooperative sensing and sensing scheduling have not been considered, whereas we jointly optimize them to minimize the overall sensing overhead.

Despite all these efforts, an important but unexplored issue is the joint exploitation of sensor selection and sensing scheduling in MAC-layer spectrum sensing to synergistically improve detection performance. We argue that 


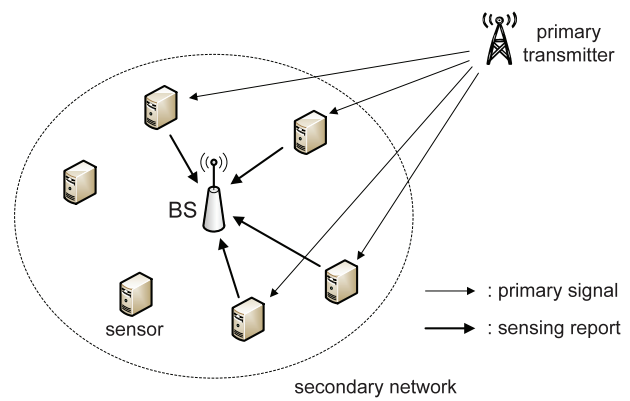

Fig. 1. DSA network model. A (sub)set of SUs (sensors) perform spectrum sensing simultaneously during each sensing period and report their measurement results to the fusion center.

investigating the interplay between the two MAC-layer sensing methods is key in devising optimal and efficient sensing schemes in DSA networks.

\section{System Model}

In this section, we introduce the DSA network model, PHYand MAC-layer spectrum sensing for incumbent detection, and assumptions that we use throughout the paper.

\subsection{DSA Network Model}

We consider a DSA network in which primary and secondary systems coexist in the same geographical area, as shown in Fig. 1. We assume a large-scale stationary primary system, such as DTV users in TV spectrum bands. A secondary system is an infrastructure-based network, e.g., IEEE 802.22 WRAN, where each cell consists of a single base station (or fusion center) and multiple stationary sensors. ${ }^{3}$ The fusion center selects a (sub)set of sensors and directs them to perform sensing by scheduling (one or more) sensing (quiet) periods. At the end of each sensing period, sensors report their measurement results to the fusion center sequentially. Based on the sensing reports collected from the sensors, the fusion center will make a final decision on the presence or absence of a primary signal and will announce the decision to the SUs in the cell. We assume the existence of a separate control channel, which provides reliable communication between the fusion center and sensors.

\subsection{Spectrum Sensing Model}

\subsubsection{PHY-Layer Spectrum Sensing}

Spectrum sensing consists of PHY- and MAC-layer mechanisms. For PHY-layer sensing, we assume the use of energy detection instead of other sensing techniques, such as matched-filter detection [42], cyclostationary feature detection [43] and compressed sensing [44]. The energy detection is one of the most widely used sensing techniques because of its simple design and low complexity; it simply measures signal power on a target frequency band and does not require a priori knowledge of primarysignal-specific features.

Regarding the existence of a primary signal on a given channel, there are two hypotheses, i.e.,

3. Although we focus here on a DSA network of stationary sensors, e.g., CPEs in the IEEE 802.22 WRANs, our sensing scheduling algorithm can be applied to mobile sensors as long as their sensing reports are not closely correlated, e.g., the sensors do not move together in a group.

$$
y_{i}(n)= \begin{cases}w_{i}(n) & \mathcal{H}_{0} \text { (no primary signal) } \\ s_{i}(n)+w_{i}(n) & \mathcal{H}_{1} \text { (primary signal exists), }\end{cases}
$$

where $y_{i}(n)$ is the signal received by a sensor, $s_{i}(n)$ is the primary signal, and $w_{i}(n)$ is an independent and identically distributed (i.i.d.) additive white Gaussian noise (AWGN) at sensor $i$ in the $n$th time slot within the sensing duration. The test statistic of the energy detector is an estimate of average RSS [13]:

$$
T_{i}=\frac{B}{M} \sum_{n=1}^{M} y_{i}(n) * y_{i}(n),
$$

where $B$ is the channel bandwidth, and $M$ is the number of signal samples during a sensing period. The measurement study in [13] indicates that the test statistic in (1) can be approximated as a Gaussian distribution using the central limit theorem (CLT) because the signal sample size, $M$, is sufficiently large, even with a short sensing duration (e.g., $1 \mathrm{~ms}$ ). For example, assuming that the signal is sampled at the Nyquist rate, a sensor can obtain $M=6 \times 10^{3}$ samples for a 6-MHz TV channel within $1 \mathrm{~ms}$ [45].

Then, the probability density function (p.d.f.) of the test statistic $T_{i}$ at sensor $i$ can be accurately approximated as [13]:

$$
T_{i} \sim \begin{cases}\mathcal{N}\left(N B, \frac{(N B)^{2}}{M}\right) & \mathcal{H}_{0} \\ \mathcal{N}\left(P_{i}+N B, \frac{\left(P_{i}+N B\right)^{2}}{M}\right) & \mathcal{H}_{1},\end{cases}
$$

where $P_{i}$ is the received primary signal strength and $N$ is the noise spectral density. Note that this Gaussian approximation is valid even in very low-SNR environments.

We make two additional assumptions as follows:

- A1. Separation between the primary transmitter and sensors is relatively larger than the separation between sensors, which is reasonable in large-scale networks such as the IEEE 802.22 WRANs [46]. ${ }^{4}$

- A2. The impact of multipath fading on spectrum sensing is negligible due to a wide channel bandwidth (e.g., larger than the coherent bandwidth) as in DTV signal detection in IEEE 802.22 WRANs [13]. ${ }^{5}$

Based on the above assumptions, the received primary signal strength at sensor $i$ can be expressed as $P_{i}=P_{R} \cdot e^{Y_{i}}$, where $P_{R}$ is the average RSS within a cell, and $e^{Y_{i}}$ is the shadowing gain between the primary transmitter and sensor $i$. Shadow fading can be characterized by the shadowing dB-spread, $\sigma_{d B}$, and it has the relationship $\sigma=0.1 \ln (10) \sigma_{d B}{ }^{6}$

Remark. It is important to note that in stationary secondary systems, where sensors do not move, the shadowing gain $Y_{i}$ is a specific realization of a normal random variable $Y \sim \mathcal{N}\left(0, \sigma^{2}\right)$. Thus, the channel gain is also (pseudo)

4. The detection of small-scale PUs, such as wireless microphones, is outside the scope of this paper.

5 . The performance of the energy detector under multipath fading can be found in [47].

6. We assume that all sensors within a secondary cell experience the same path-loss rate since the relative distance to the primary transmitter is much larger than the distances between the sensors [46] 
time invariant and determined based on sensor locations. We will elaborate further on temporal correlation in sensing reports among station sensors in Section 5.1.

\subsubsection{MAC-Layer Spectrum Sensing}

The performance of energy detection is highly susceptible to signal-to-noise ratio (SNR), thus limiting its ability to meet the FCC's detection requirements. Moreover, noise uncertainty [48] at sensors also prevents the applicability of energy detection to such a weak primary signal. As a result, one-time sensing with a single sensor cannot guarantee the detection requirements imposed by regulatory bodies such as the FCC.

To overcome these challenges, we design efficient MAClayer spectrum sensing algorithms-i.e., sensor selection and sensing scheduling - by exploiting both spatial diversity and temporal variations in sensing reports (i.e., RSS) to improve the incumbent detection performance. Most previous work studies these two MAC-layer sensing methods separately; therefore, the analysis of their interplay is still an open problem.

Once the fusion center collects sensing reports (i.e., measured RSSs) from sensors, it will perform data fusion to make a decision. As we discuss next, we propose to build an RSS profile for data fusion.

\section{Data-Fusion Rule Based on Spatial RSS Profile}

In this section, we discuss the design rationale behind our approach, present the construction of a spatial RSS profile, and formulate the problem of incumbent detection based on one-time sensing as a binary classification problem via LDA. We then analyze its detection performance and further characterize the wireless network conditions, under which more sensing is needed to meet desired detection requirements.

\subsection{Design Rationale}

As mentioned earlier, in DSA networks with stationary sensor deployment, the measured RSS at each sensor is (pseudo) time invariant. This allows the fusion center to learn the RSS distributions at sensors and construct their spatial RSS profile. The fusion center can construct a unique profile of RSS distributions for a given set of sensors and sensing time. For RSS profiling, we assume a large enough training period (including both ON/OFF periods of the primary transmitter) for accurate estimation of RSS distributions. In practice, the RSS profile can be constructed at the time of system/sensor deployment.

Upon collecting the sensing results during each scheduled sensing period, the fusion center compares the observed RSS values with the RSS profile. A similarity between the RSS distribution and the primary signal can be interpreted as an indication of the presence of a primary signal, and vice versa. Using the RSS profile, the fusion center can adopt the sequential hypothesis testing framework for sensing scheduling, and minimally schedule sensing periods only until it accumulates a sufficient number of observations to determine whether or not a primary signal exists within a certain performance bound.

The RSS-profile-based data fusion offers two main benefits: 1) near-optimal detection performance, and 2) high robustness (fault tolerance) against attacks (malfunctioning sensors). We will demonstrate the above benefits of the proposed RSS-profile-based spectrum sensing in Section 6.

\subsection{Construction of a Spatial RSS Profile}

We propose to build a spatial profile of RSSs at multiple sensor locations, which will be used as a main reference for incumbent detection. To build a RSS profile, it is important to understand the spatial and temporal characteristics of sensing reports. The spatial RSS diversity is due mainly to the different sensors' locations (thus different channel gains from the primary transmitter), whereas the temporal RSS variations are due mainly to the measurement error of the energy detector. The intensity of temporal variations depends on the sensing time (i.e., $M$ in (1)); the shorter the sensing time, the larger the temporal RSS variation due to the increase of measurement error.

Note that the measurement study from the IEEE 802.22 Working Group indicated that the distribution of test statistic, $T$ (equivalent to the estimated RSS), of the energy detector can be approximated as Gaussian (see (2)) using the CLT in both ON and OFF periods. Thus, the RSS profile of $n_{s}$ stationary sensors is an $n_{s}$-dimensional Gaussian distribution, the parameters of which can be easily estimated using well-known techniques, such as maximum-likelihood estimation (MLE). Since the RSS distributions at each sensor location are (pseudo) time invariant due to the static deployment of SUs, the RSS profile can be used reliably without frequent updating.

\subsection{Detection with One-Time Sensing Based on LDA}

We now present a detection rule using an RSS profile given a one-time sensing measurement. Let $\mathbf{x}=\left[T_{1}, \ldots, T_{n_{s}}\right]^{T}$ denote the vector of test statistics of the energy detector measured by $n_{s}$ cooperating sensors. Then, the incumbent detection problem can be cast into a binary Gaussian classification problem, where the observed test statistic $\mathrm{x} \in$ $\mathbb{R}^{n_{s} \times 1}$ belongs to one of two classes, $\mathcal{H}_{0}$ or $\mathcal{H}_{1}$, where

$$
\begin{array}{ll}
\mathcal{H}_{0}: \mathrm{x} \sim \mathcal{N}\left(\mu_{0}, \Sigma_{0}\right) & \text { (no primary signal) } \\
\mathcal{H}_{1}: \mathrm{x} \sim \mathcal{N}\left(\mu_{1}, \Sigma_{1}\right) & \text { (primary signal exists) }
\end{array}
$$

where $\boldsymbol{\mu}_{k} \in \mathbb{R}^{n_{s} \times 1}$ and $\Sigma_{k} \in \mathbb{R}^{n_{s} \times n_{s}}$ are the estimated mean vector and the covariance matrix of RSS distributions under $\mathcal{H}_{k}$, respectively. Note that $\Sigma_{0}=\sigma_{n}^{2} \mathbf{I}$, where $\mathbf{I}$ is an $n_{s} \times n_{s}$ identity matrix and $\sigma_{n}^{2}=(N B)^{2} / M$.

Under the general assumption of unequal covariance matrices, i.e., $\Sigma_{0} \neq \Sigma_{1}$, the optimal decision rule for our detection problem can be found via quadratic discriminant analysis (QDA) [49]. Although QDA provides optimal detection performance for a general multivariate Gaussian with unequal covariance matrices, its quadratic decision boundaries do not yield a closed-form expression for detection performance [50].

In our problem, the quadratic decision rule can actually be linearized using a LDA on the basis of the following two important observations, i.e., the covariance matrix under $\mathcal{H}_{1}, \Sigma_{1}$, can be:

- O1) assumed as an identity matrix with fixed sensor locations, and then,

- O2) approximated as $\Sigma_{1} \approx \Sigma_{0}=\sigma_{n}^{2} \mathbf{I}$ in a very low $S N R$ environment. 


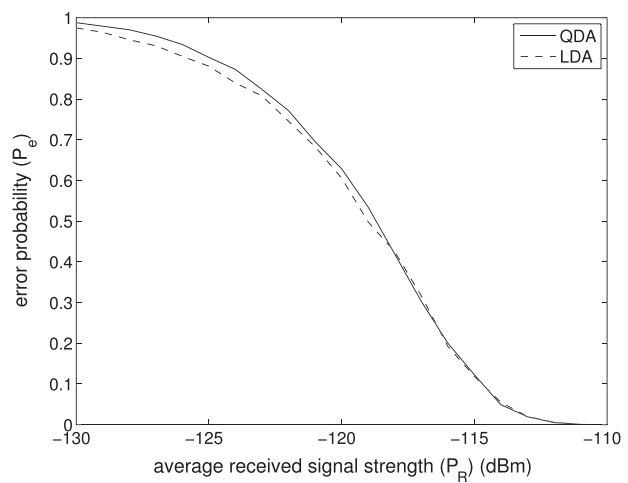

Fig. 2. Error performances of QDA versus LDA. The performance difference is insignificant, even in a very low SNR environment. These are the results of a Monte Carlo simulation with $10^{7}$ runs.

Regarding the first observation, the covariance matrix $\Sigma_{1}$ may not appear to be an identity matrix because of the existence of shadow correlation in primary signal strengths [51]. However, as mentioned earlier, when sensor locations are fixed, their RSSs are also (pseudo) time invariant and the randomness in the test statistics comes only from the noise processes (i.e., measurement errors), which are independent of each other. Thus, the correlation of RSSs between any pair of sensors does not exist, so we can assume that $\Sigma_{1}$ is also an identity matrix as $\Sigma_{0}$ (see Section 5.1).

Regarding the second observation, the received primary signal strength may be significantly lower than that of the noise power for a very weak primary signal. For example, the FCC requires the detection of a DTV signal as weak as $-20 \mathrm{~dB}$, assuming the typical noise level $N B=-95.2 \mathrm{dBm}$ [14]. Therefore, it is reasonable to assume that $P_{i}+N B \approx$ $N B \forall i$, and thus, $\Sigma_{1} \approx \Sigma_{0}=\sigma_{n}^{2}$ I. Fig. 2 justifies these assumptions by showing that the error performances of QDA and LDA are almost the same in very low SNR environments.

Lemma 1. With spatial RSS profile, we have a simple distancebased decision rule for incumbent detection:

$$
\left\|\mathbf{x}-\mu_{0}\right\| \underset{\mathcal{H}_{1}}{\stackrel{\mathcal{H}_{0}}{\gtrless}}\left\|\mathbf{x}-\mu_{1}\right\|,
$$

where $\mathbf{x}$ is the measured RSS vector, and $\boldsymbol{\mu}_{k}$ is the estimated mean vector of the RSS at sensors under $\mathcal{H}_{k}$.

Proof. Based on the observations O1) and O2), we compute the log likelihood of the two hypotheses $\mathcal{H}_{0}$ and $\mathcal{H}_{1}$ as:

$$
\begin{aligned}
\log \left(\frac{\operatorname{Pr}\left(\mathcal{H}_{1} \mid \mathbf{x}\right)}{\operatorname{Pr}\left(\mathcal{H}_{0} \mid \mathbf{x}\right)}\right)= & \log \frac{g_{1}(\mathbf{x})}{g_{0}(\mathbf{x})}+\log \frac{\pi_{1}}{\pi_{0}} \\
= & \log \frac{\pi_{1}}{\pi_{0}}-\frac{1}{2}\left(\boldsymbol{\mu}_{1}+\boldsymbol{\mu}_{0}\right)^{T} \boldsymbol{\Sigma}^{-1}\left(\boldsymbol{\mu}_{1}-\boldsymbol{\mu}_{0}\right) \\
& +\mathbf{x}^{T} \boldsymbol{\Sigma}^{-1}\left(\boldsymbol{\mu}_{1}-\boldsymbol{\mu}_{0}\right),
\end{aligned}
$$

where $g_{k}(\mathbf{x})$ is the estimated Gaussian distribution of the sensing reports, $\mathbf{x}$, under $\mathcal{H}_{k}$ and $\Sigma$ is the common covariance matrix. $\pi_{k}$ is a priori probabilities of hypotheses $\mathcal{H}_{k}$.

Then, in our two-class problem, the fusion center will assume $\mathcal{H}_{1}$ if the following condition holds:

$$
\mathbf{x}^{T} \Sigma^{-1}\left(\hat{\boldsymbol{\mu}}_{1}-\hat{\boldsymbol{\mu}}_{0}\right)>\frac{1}{2} \hat{\boldsymbol{\mu}}_{1}^{T} \hat{\boldsymbol{\Sigma}}^{-1} \hat{\boldsymbol{\mu}}_{1}-\frac{1}{2} \hat{\boldsymbol{\mu}}_{0}^{T} \hat{\boldsymbol{\Sigma}}^{-1} \hat{\boldsymbol{\mu}}_{0}+\log \frac{\pi_{0}}{\pi_{1}} .
$$

Otherwise, the fusion center will assume $\mathcal{H}_{0}$. Assuming that the fusion center may not have an accurate estimation of a priori probability of a primary signal, the fusion center can set $\pi_{0}=\pi_{1}=0.5$. Then, (3) follows.

Equation (3) indicates that, under both hypotheses, the decision is made based solely on the distance between the observed RSS vector, $x$, and the mean vectors of the RSS profile, $\boldsymbol{\mu}_{k}$. Although (3) is optimal in minimizing detection error performance (i.e., the sum of false alarm and misdetection probabilities), the detection requirements are often expressed in terms of misdetection probability for a fixed false-alarm probability. In what follows, we analyze the performance of the proposed RSS-profile-based spectrum sensing.

\subsection{Performance Analysis}

\subsubsection{Incumbent Detection Performance}

Let $\mathcal{T}(\mathbf{x}) \triangleq \mathbf{w}^{T} \mathbf{x}$ denote the test statistic for incumbent detection, which is calculated based on the observed RSS vector $\mathbf{x}$, where $\mathbf{w} \triangleq\left(\mu_{1}-\mu_{0}\right) \in \mathbb{R}^{n_{s} \times 1}$. Note that $\|\mathbf{w}\|$ is the euclidean distance between the centroids of two Gaussian distributions under both hypotheses, where the centroids are the vectors of average RSSs at sensor locations. It can be easily shown that the test statistic $\mathcal{T}(\mathbf{x})$ follows a Gaussian distribution, i.e., $\mathcal{T}(\mathbf{x}) \sim \mathcal{N}\left(\mathbf{w}^{T} \boldsymbol{\mu}_{k}, \sigma_{n}^{2}\|\mathbf{w}\|^{2}\right)$ under $\mathcal{H}_{k}$.

Then, the probability of false alarm under our LDA-based decision rule with the decision threshold $\eta \in \mathbb{R}$ is given as:

$$
P_{F A}^{L D A} \triangleq \operatorname{Prob}\left(\mathcal{T}(\mathbf{x})>\eta \mid \mathcal{H}_{0}\right)=Q\left(\frac{\eta-\mathbf{w}^{T} \boldsymbol{\mu}_{0}}{\sigma_{n}\|\mathbf{w}\|}\right),
$$

where $Q(\cdot)$ is the Q-function. Using (4), the decision threshold $\eta$ can be derived for the desired $P_{F A}^{L D A}$ as:

$$
\eta=\sigma_{n} \cdot\|\mathbf{w}\| \cdot Q^{-1}\left(P_{F A}^{L D A}\right)+\mathbf{w}^{T} \boldsymbol{\mu}_{0} .
$$

Then, based on (4) and (5), the probability of misdetection, $P_{M D}^{L D A}$, is given as:

$$
\begin{aligned}
P_{M D}^{L D A} & \triangleq \operatorname{Prob}\left(\mathcal{T}(\mathbf{x})<\eta \mid \mathcal{H}_{1}\right) \\
& =1-Q\left(Q^{-1}\left(P_{F A}^{L D A}\right)-\frac{\|\mathbf{w}\|}{\sigma_{n}}\right) .
\end{aligned}
$$

Equation (6) indicates that, when the desired false-alarm probability, $P_{F A}^{L D A}$, is given, the achievable misdetection, $P_{M D}^{L D A}$, depends on the noise variance $\sigma_{n}^{2}=\frac{(N B)^{2}}{M}$ in energy detection and the distance $\|\mathbf{w}\|$. That is, $P_{M D}^{L D A}$ decreases as the sensing duration (thus, the number $M$ of sensing samples) increases because a large number of samples would make the decision more accurate due to the reduced noise variance (measurement error).

\subsubsection{Impact of Shadow Fading}

Recall that $\mathrm{w}$ is defined as the difference in RSSs under both hypotheses, i.e., $\mathbf{w} \triangleq\left(\mu_{1}-\mu_{0}\right)=\left[P_{1}, \ldots, P_{n_{s}}\right]^{T}$. Therefore, $\|\mathbf{w}\|$ under shadow fading is given as:

$$
\|\mathbf{w}\|=\left[\sum_{i=1}^{n_{s}} P_{i}^{2}\right]^{1 / 2}=P_{R} \cdot\left[\sum_{i=1}^{n_{s}}\left(e^{Y_{i}}\right)^{2}\right]^{1 / 2},
$$



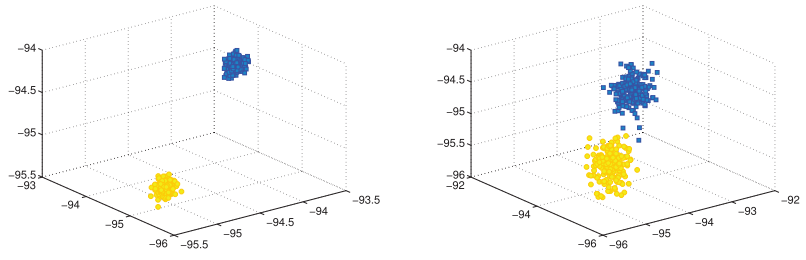

(a) $P_{R}=-100 \mathrm{dBm}, T_{S}=1 \mathrm{~ms}$

(b) $P_{R}=-100 \mathrm{dBm}, T_{S}=100 \mu \mathrm{s}$
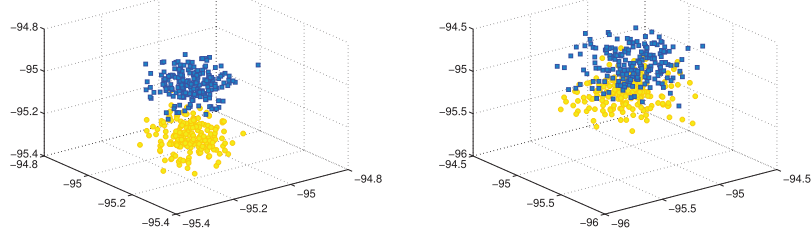

(c) $P_{R}=-110 \mathrm{dBm}, T_{S}=1 \mathrm{~ms}$

(d) $P_{R}=-110 \mathrm{dBm}, T_{S}=100 \mu \mathrm{s}$

Fig. 3. Distribution of the measured RSS vector from three cooperating sensors under various wireless environments. The yellow (blue) dots indicate the vector of measured test statistics under $\mathcal{H}_{0}\left(\mathcal{H}_{1}\right)$. The simulation parameters are set $N B=-95.2 \mathrm{dBm}$ and $\sigma_{d B}=5.5 \mathrm{~dB}$.

where $P_{R}$ is the average RSS in the secondary cell due to path loss and $Y_{i}$ is a location-dependent realization of a random variable $Y \sim \mathcal{N}\left(0, \sigma^{2}\right)$ where $\sigma=0.1 \ln (10) \sigma_{d B}$.

To understand the impact of shadow fading on detection performance (in terms of $P_{M D}^{L D A}$ given a fixed $P_{F A}^{L D A}$ ), we study the distribution of $\|\mathbf{w}\|$ in (7). Although there is no closed-form expression available for the power sum of lognormal random variables in (7) [52], the power sum can be approximated accurately by rendering the sum itself as another log-normal random variable [53].

Let $e^{Z^{\prime}} \sim e^{2 Y_{1}}+e^{2 Y_{2}}+\cdots+e^{2 Y_{n_{s}}}$. Then, by following the result in [53], the sum can be approximated by matching its mean and variance with $e^{Z^{\prime}}$. The first two moments of $e^{Z^{\prime}}$ are $\mathbb{E}\left[e^{Z^{\prime}}\right]=e^{\mu_{Z^{\prime}}+\sigma_{Z^{\prime}}^{2} / 2}$ and $\mathbb{E}\left[e^{2 Z^{\prime}}\right]=e^{2 \mu_{Z^{\prime}}+2 \sigma_{Z^{\prime}}^{2}}$. Our final goal is to approximate the square root of the power sum, i.e., $e^{Z}=\left(e^{Z^{\prime}}\right)^{1 / 2}$, which is still a log-normal random variable. Thus, by equating the first two moments of $e^{Z^{\prime}}$ and the power sum, $\sum_{i=1}^{n_{s}}\left(e^{Y_{i}}\right)^{2}$, and then taking $e^{Z}=\left(e^{Z^{\prime}}\right)^{1 / 2}$, we have $\|\mathbf{w}\| \approx P_{R} \cdot e^{Z}$ with the random variable $e^{Z} \sim$ $\log -\mathcal{N}\left(\mu_{Z}, \sigma_{Z}^{2}\right)$ where:

$$
\sigma_{Z}^{2}=\frac{1}{4} \log \left[\frac{\left(e^{4 \sigma^{2}}-1\right)}{n_{s}}+1\right]
$$

and

$$
\mu_{Z}=\frac{1}{2} \log \left(n_{s}\right)+\sigma^{2}-\frac{\sigma_{Z}^{2}}{4} .
$$

Then, we can derive the average misdetection probability as:

$$
\bar{P}_{M D}^{L D A}=\int_{-\infty}^{\infty}\left[1-Q\left(Q^{-1}\left(P_{F A}^{L D A}\right)-\frac{P_{R} \cdot e^{z}}{\sigma_{n}}\right)\right] \cdot f_{z} \cdot \mathrm{d} z,
$$

where

$$
f_{z}=\frac{1}{\sigma_{Z} \sqrt{2 \pi}} \exp \left[-\frac{\left(z-\mu_{Z}\right)^{2}}{2 \sigma_{Z}^{2}}\right],-\infty<z<\infty .
$$

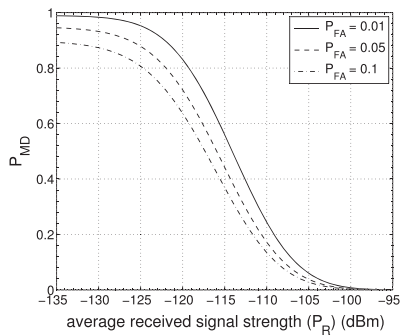

Fig. 4. Detection performance of RSS-profile-based cooperative spectrum sensing for various primary signal strengths.

Fig. 3 shows examples of RSS distributions under various wireless environments. The figure shows that the centroids of the two Gaussian distributions will have a larger separation, $\|\mathbf{w}\|$, with a higher average primary signal strength $P_{R}$, or a longer sensing time $T_{S}$. As indicated in (6), a larger separation between the two RSS distributions improves the incumbent detection performance.

Fig. 4 plots misdetection probabilities, $P_{M D}$, for a given false-alarm probability. The figure shows that the misdetection probability increases as the average primary signal strength decreases. It shows that spectrum sensing with a single sensor may not be sufficient to protect primary communications. To further improve the detection performance while introducing minimal sensing overhead, in what follows, we jointly optimize sensor selection, sensing time, and sensing scheduling.

\subsection{The Necessity of Sensing Scheduling}

We now characterize the network conditions under which the fusion center must schedule spectrum sensing multiple times to meet a given detectability requirement. As we observed in previous sections, performance of one-time spectrum sensing depends on various network parameters, such as average primary signal strength, $P_{R}$, number of sensors for sensing collaboration $n_{s}$, and detection requirements, $P_{F A}$ and $P_{M D}$. The fusion center may avoid scheduling sensing multiple times by employing a larger number of sensors for cooperative sensing. We define the minimum number of sensors, $n_{s}^{*}$, to meet a given detection requirement as:

$$
n_{s}^{*} \triangleq \arg \min _{n_{s}}\left\{n_{s}: \bar{P}_{F A}, \bar{P}_{M D} \leq \xi\right\} .
$$

Fig. 5 plots the minimum number of sensors required to achieve the desired level of detection probability, $\xi$. This figure shows that the required number of sensors increases exponentially as the average primary signal

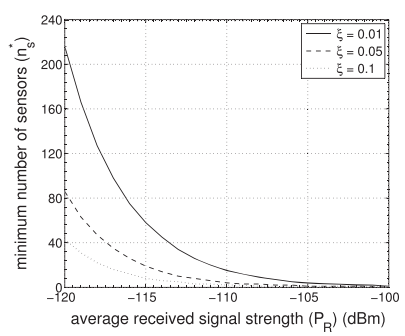

Fig. 5. Minimum number of sensors required to achieve a desired detection performance with one-time sensing. 


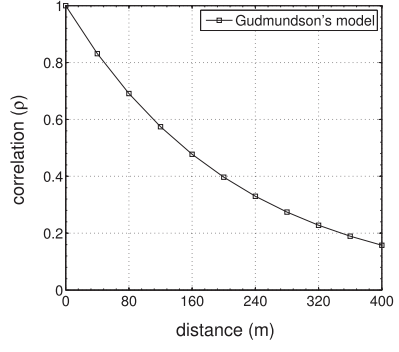

(a) spatial domain

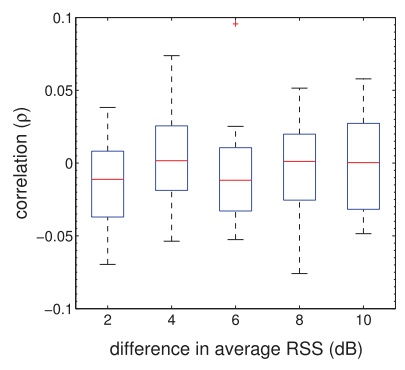

(b) temporal domain
Fig. 6. Spatial versus temporal correlation in shadow fading: (a) the spatial shadowing correlation decreases with increasing separation between sensors where $d_{\text {corr }}=150 \mathrm{~m}$, whereas (b) the temporal correlation in sensing reports is virtually zero regardless of the difference in average RSS at sensors.

strength, $P_{R}$, decreases. The number of sensors also increases as the detection requirement becomes stricter, i.e., smaller $\xi$. For example, when $P_{R}=-116 \mathrm{dBm}$, more than 70 sensors are required to achieve $P_{F A}, P_{M D} \leq 0.01$. In practice, it may be difficult to find such a large number of sensors within a cell, and more importantly, having such a large number of sensors may incur significant overhead. Therefore, in a low SNR environment with strict detection requirements, the fusion center must schedule sensing multiple times to make the best tradeoff between performance and overhead.

\section{Joint Optimal Design of Cooperative Sensing and Sensing Scheduling}

In this section, we first investigate temporal correlation in sensing reports among stationary sensors. We then propose adaptive online algorithms that find: 1) an optimal stopping time for scheduling sensing periods; 2) an optimal set of sensors; and 3) an optimal sensing duration that minimizes the average sensing-time overhead.

\subsection{Temporal Correlation in Sensing Reports}

Before presenting an optimal sensing scheduling algorithm, we would like to show that there is no correlation in RSSs among stationary sensors. As we discussed in Section 4.3, the randomness in sensing results (i.e., the output of the energy detector) comes only from the measurement noise, which is independent for each sensor. To illustrate this, we measure the Pearson's correlation coefficient between a pair of sensors. The Pearson's correlation coefficient of sensing reports from the sensors $i$ and $j$ can be calculated as:

$$
\hat{\rho}_{i, j}=\operatorname{Corr}(i, j)=\frac{\sum_{i=1}^{n}\left(T_{i}-\mathbb{E}\left[T_{i}\right]\right)\left(T_{j}-\mathbb{E}\left[T_{j}\right]\right)}{(n-1) \hat{\sigma}_{i} \hat{\sigma}_{j}},
$$

where $\hat{\sigma}_{i}$ and $\hat{\sigma}_{j}$ are the sample standard deviation of test statistics $T_{i}$ and $T_{j}$, i.e.,

$$
\hat{\sigma}_{i} \triangleq\left(\frac{1}{n-1} \sum_{i=1}^{n}\left(T_{i}-\mathbb{E}\left[T_{i}\right]\right)^{2}\right)^{\frac{1}{2}} .
$$

It is well known that shadow fading is correlated with the spatial domain [51], as shown in Fig. 6a. As a result, sensors located close to each other (e.g., within shadowing decorrelation distance) are highly likely to exhibit similar

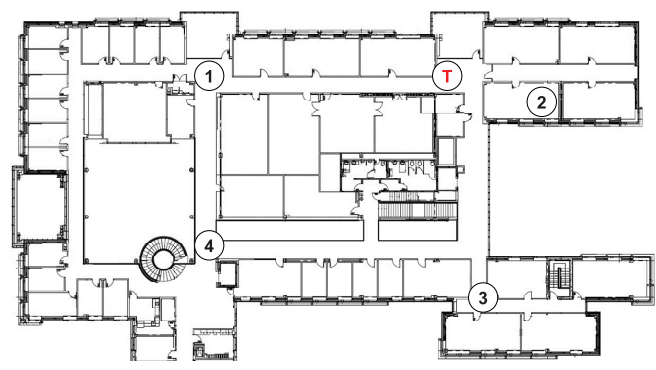

Fig. 7. GNURadio testbed on the fourth floor of the CSE building at the University of Michigan. It consists of one transmitter (denoted as T) and four receivers (1-4) working as spectrum sensors.

sensing reports. However, Fig. $6 \mathrm{~b}$ indicates that even when sensors are located close to each other (and thus they have similar average RSS values), their sensing reports are not correlated with the temporal domain.

\subsection{Experimental Results}

To examine the temporal correlation in RSS in a realistic network environment, we measured RSS at four different locations in our GNUradio/USRP2 [54] testbed deployed on the fourth floor of the Computer Science and Engineering (CSE) Building at the University of Michigan. We placed five USRP2 nodes (one transmitter and four receivers) at different locations (i.e., corridors and offices) shown in Fig. 7. We emulate the DSA network by considering the transmitter and the receivers as the primary transmitter and the spectrum sensors, respectively. We equipped the nodes with the VERT2450 (dual Band $2,400-2,480 \mathrm{MHz}$ and $4.9-5.9-\mathrm{GHz}$ omnidirectional antenna) on a XCVR2450 board (2.4-2.5 and 4.9-5.85-GHz dual-band daughterboard).

We measured the SNR at the receivers (thus emulating the energy detector) for 1-min periods, as shown in Fig. 8a. The SNR was found to be very stable during the measurement periods, except for the measurement noise. Fig. 8b shows the correlation coefficient in the measured time series of SNRs at six receiver pairs. The results show that the correlation coefficients are negligible for the receiver pairs, indicating that there is virtually no correlation with the measured SNR when the sensors are stationary.

The measurement results imply that when the fusion center can obtain the information of average primary signal strength at each sensor (via estimation or learning), then the physical separation between cooperating sensors may not

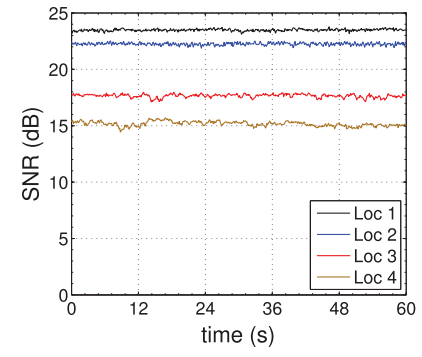

(a) SNR

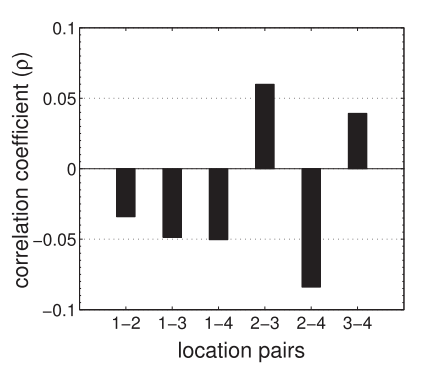

(b) correlation

Fig. 8. Temporal correlation in measured RSS among stationary sensors. (a) The measured SNR is stable at different locations, and (b) the correlation among the measured SNR is negligible (i.e., $\rho<0.1$ ). 


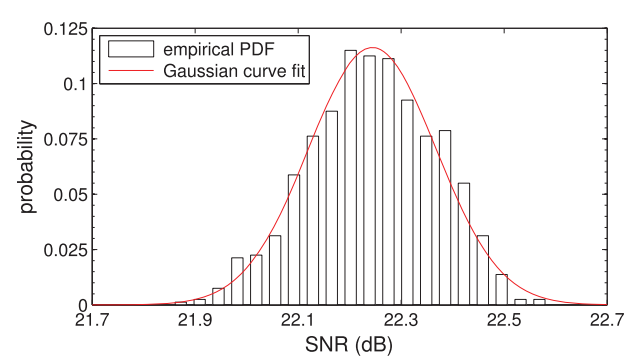

Fig. 9. The measured SNR can be approximated as a Gaussian distribution.

greatly affect detection performance. ${ }^{7}$ These uncorrelated sensing reports in the temporal domain allow us to use the sequential hypothesis testing framework to find the optimal time to stop the scheduling of sensing as we discuss next. Moreover, the results also indicate that the measured signal strengths (or sensing results) can be approximated well as a Gaussian distribution, as shown in Fig. 9, corroborating our assumption in Section 3.

\subsection{Optimal Stopping Rule for Sensing Scheduling}

The fusion center can schedule sensing multiple times before making a final decision, and thus, it receives a sequence of sensing reports from the sensors, which are independent of each other.

This makes sequential detection suitable for our problem. In particular, among the various sequential detection schemes, we adopt Wald's SPRT [55] because it is optimal in the sense of minimizing the average number of observations, given bounded probabilities of false alarm and misdetection.

Let $t_{n} \triangleq \sigma_{n}^{-1} \cdot\|\mathbf{w}\|^{-1} \cdot \mathcal{T}\left(\mathbf{x}_{n}\right)$ denote the normalized test statistic based on the observed RSS vector $\mathbf{x}_{n}$ in the $n$th sensing period. The decision statistic $\Lambda_{N}$ is the log-likelihood ratio based on $N$ sequential observations (i.e., test statistics) $t_{1}, \ldots, t_{N}$ as:

$$
\Lambda_{N} \triangleq \lambda\left(t_{1}, \ldots, t_{N}\right)=\ln \frac{f_{1}\left(t_{1}, \ldots, t_{N}\right)}{f_{0}\left(t_{1}, \ldots, t_{N}\right)},
$$

where $f_{k}\left(t_{1}, \ldots, t_{N}\right)$ is the joint p.d.f. of the sequence of observations under hypotheses $\mathcal{H}_{k} \forall k$. Recall that $\left\{t_{n}\right\}_{n=1}^{N}$ are Gaussian, and they are independent, as we observed in Section 5.1. Then, (13) becomes:

$$
\Lambda_{N}=\sum_{n=1}^{N} \lambda_{n}=\sum_{n=1}^{N} \ln \frac{f_{1}\left(t_{n}\right)}{f_{0}\left(t_{n}\right)},
$$

where $f_{k}\left(t_{n}\right)$ is $\mathcal{N}\left(\theta_{k}, 1\right)$ with $\theta_{k} \triangleq \mathbb{E}\left[t_{n} \mid \mathcal{H}_{k}\right]=\frac{\mathbf{w}^{T} \mu_{k}}{\sigma_{n}\|\mathbf{w}\|} \forall k$. Then, we have:

$$
\lambda_{n}=\ln \frac{f_{1}\left(t_{n}\right)}{f_{0}\left(t_{n}\right)}=\left(\theta_{1}-\theta_{0}\right) t_{n}+\frac{1}{2}\left(\theta_{0}^{2}-\theta_{1}^{2}\right) .
$$

Based on (14) and (15), the decision statistic $\Lambda_{N}$ can be expressed as:

7. Note that, in a stationary DSA network environment, such as the IEEE 802.22 WRAN where all the sensors (called CPEs) in the network do not move, the fusion center can easily obtain the RSS at each sensor, e.g., by simply observing the reports from the sensors.

$$
\Lambda_{N}=\left(\theta_{1}-\theta_{0}\right) \sum_{n=1}^{N} t_{n}+\frac{N}{2}\left(\theta_{0}^{2}-\theta_{1}^{2}\right) .
$$

Then, in SPRT, a decision is made based on the observed sequence of test statistics, $\left\{t_{n}\right\}_{n=1}^{N}$, using the following rule:

$$
\begin{aligned}
\Lambda_{N} \geq \Gamma_{u} & \Rightarrow \text { accept } \mathcal{H}_{1} \text { (primary signal exists) }, \\
\Lambda_{N}<\Gamma_{l} & \Rightarrow \text { accept } \mathcal{H}_{0} \text { (no primary signal), } \\
\Gamma_{l} \leq \Lambda_{N}<\Gamma_{u} & \Rightarrow \text { take another observation, }
\end{aligned}
$$

where $\Gamma_{l}$ and $\Gamma_{u}\left(0<\Gamma_{l}<\Gamma_{u}<\infty\right)$ are the detection thresholds that depend on the desired values of $P_{F A}$ and $P_{M D}$.

Let $\alpha^{*}$ and $\beta^{*}$ denote the desired values of false-alarm and misdetection probabilities, respectively. Then, the decision boundaries are given by Wald [55]:

$$
\Gamma_{l}=\ln \frac{\beta^{*}}{1-\alpha^{*}} \quad \text { and } \quad \Gamma_{u}=\ln \frac{1-\beta^{*}}{\alpha^{*}} .
$$

\subsection{Sensing Delay Analysis}

Recall that our goal is to minimize the number of times the spectrum needs to be sensed, with the decision thresholds derived from the target detection probabilities as shown in (17). We, therefore, consider the number of sensing periods scheduled until a decision is made (i.e., either the boundary $\Gamma_{l}$ or $\Gamma_{u}$ is reached) as our main performance metric. The average number of sensing periods, $\mathbb{E}[N]$, required for decision making can be computed as:

$$
\mathbb{E}\left[\Lambda_{N}\right]=\mathbb{E}[N] \times \mathbb{E}\left[\lambda \mid \mathcal{H}_{k}\right] .
$$

First, using (15), the average value of $\lambda$ under $\mathcal{H}_{k}$ can be derived as:

$$
\mathbb{E}\left[\lambda \mid \mathcal{H}_{k}\right]=\left(\theta_{1}-\theta_{0}\right) \theta_{k}+\frac{1}{2}\left(\theta_{0}^{2}-\theta_{1}^{2}\right) .
$$

The average of $\Lambda_{N}$ can then be found as follows: Suppose $\mathcal{H}_{0}$ holds, then $\Lambda_{N}$ will reach $\Gamma_{u}$ (i.e., false alarm) with the desired false-alarm probability $\alpha^{*}$; otherwise, it will reach $\Gamma_{l}$. Thus, using (17), we have:

$$
\mathbb{E}\left[\Lambda_{N} \mid \mathcal{H}_{0}\right]=\alpha^{*} \ln \frac{1-\beta^{*}}{\alpha^{*}}+\left(1-\alpha^{*}\right) \ln \frac{\beta^{*}}{1-\alpha^{*}} .
$$

Based on (18), (19), and (20), we can derive the average required number of sensing periods for decision making as:

$$
\mathbb{E}\left[N \mid \mathcal{H}_{0}\right]=\frac{\alpha^{*} \ln \frac{1-\beta^{*}}{\alpha^{*}}+\left(1-\alpha^{*}\right) \ln \frac{\beta^{*}}{1-\alpha^{*}}}{\left(\theta_{1}-\theta_{0}\right) \theta_{0}+\frac{1}{2}\left(\theta_{0}^{2}-\theta_{1}^{2}\right)} .
$$

Similarly, we can derive:

$$
\mathbb{E}\left[N \mid \mathcal{H}_{1}\right]=\frac{\left(1-\beta^{*}\right) \ln \frac{1-\beta^{*}}{\alpha^{*}}+\beta^{*} \ln \frac{\beta^{*}}{1-\alpha^{*}}}{\left(\theta_{1}-\theta_{0}\right) \theta_{1}+\frac{1}{2}\left(\theta_{0}^{2}-\theta_{1}^{2}\right)} .
$$

Based on (13)-(22), Algorithm 1 describes our online algorithm for scheduling sensing periods that finds the optimal stopping time for sensing.

\section{Algorithm 1. ONLINE SENSING SCHEDULING.}

The fusion center does the following

1: while each round $n \in\left[1, N_{\max }\right]$ of sensing period do

2: $\quad$ Receive results of energy detector (i.e., RSS) $\mathbf{x}_{n}$ from sensors 
3: $\quad t_{n} \leftarrow \sigma_{n}^{-1} \cdot\|\mathbf{w}\|^{-1} \cdot \mathcal{T}\left(\mathbf{x}_{n}\right) / /$ Calculate test statistic

4: $\quad \Lambda_{N} \leftarrow \Lambda_{N}+\left(\theta_{1}-\theta_{0}\right) t_{n}+\frac{1}{2}\left(\theta_{0}^{2}-\theta_{1}^{2}\right)$

5: $\quad$ if $\Lambda_{N} \geq \Gamma_{u}$ then

6: $\quad$ A primary exists and we schedule fine-sensing (or initiate the channel vacation procedure)

7: $\quad$ else if $\Lambda_{N}<\Gamma_{l}$ then

8: $\quad$ A primary does not exist

9: $\quad$ else if $n==N_{\max }$ then

10: $\quad$ Schedule fine-sensing for in-depth measurement

11: else

12: $\quad$ Schedule another sensing period and wait for the

13: end if observation

14: end while

\subsection{Tradeoff in Sensing Overhead: Energy Detection versus Feature Detection}

In practice, the number of sensing periods that can be scheduled before the fusion center makes a final decision can be upper bounded by $N_{\max }$ due to several factors, such as the detection delay requirement, intersensing interval, initial sensing delay, and sensing time [56]. For example, in the 802.22 WRAN standard draft, the SUs must be able to detect the return of a PU within 2 seconds [12]. Otherwise, the fusion center must schedule a fine-sensing (i.e., feature detection) period at the end of the current sensing interval to perform expensive feature detection. Therefore, we set a threshold $P_{t h}$-a design parameter-such that the fusion center must reach a conclusion within $N_{\max }$ sensing periods with a probability greater than or equal to $P_{t h}$.

Let $N_{\text {opt }}$ denote the optimal stopping time of sensing under Algorithm 1. Then, we want to derive the probability of satisfying $N_{o p t} \leq N_{\text {max }}$, which should be no less than $P_{t h}$. Although an approximate expression for the distribution of $N_{\text {opt }}$ can be derived, we instead derive a lower bound of the probability for computational efficiency [55].

Suppose $\Lambda_{N_{\max }} \geq \Gamma_{u}$. Then, we have $N_{\text {opt }} \leq N_{\max }$, so the following inequality holds:

$$
\operatorname{Prob}\left(N_{\text {opt }} \leq N_{\max }\right) \geq \operatorname{Prob}\left(\Lambda_{N_{\max }} \geq \Gamma_{u}\right) .
$$

Since $N_{\max }$ is sufficiently large in practice, we can use the CLT, and the inequality $\Lambda_{N_{\max }} \geq \Gamma_{u}$ can then be written as:

$$
\frac{\Lambda_{N_{\max }}-N_{\max } \mathbb{E}\left[\lambda \mid \mathcal{H}_{1}\right]}{\sqrt{N_{\max }} \sigma_{1}(\lambda)} \geq \frac{B-N_{\max } \mathbb{E}\left[\lambda \mid \mathcal{H}_{1}\right]}{\sqrt{N_{\max }} \sigma_{1}(\lambda)},
$$

where $\sigma_{1}(\lambda)$ is the standard deviation of $\lambda$ under $\mathcal{H}_{1}$, which can be derived as $\sigma_{k}(\lambda)=\left(\theta_{1}-\theta_{0}\right) \forall k$ from (15). Then, the left-hand side of (24) is normally distributed with zero mean and unit variance when $\mathcal{H}_{1}$ is true.

Therefore, based on (23) and (24), we have the following lower bound of the probability that the fusion center makes a decision within $N_{\max }$ observations:

$$
\operatorname{Prob}\left(N_{\text {opt }} \leq N_{\text {max }}\right) \geq \underline{\boldsymbol{\Pi}}=\underbrace{Q\left(\frac{B-N_{\text {max }} \mathbb{E}\left[\lambda \mid \mathcal{H}_{1}\right]}{\sqrt{N_{\text {max }}} \sigma_{1}(\lambda)}\right)}_{\text {this part should be } \geq P_{\text {th }}} .
$$

This lower bound will be considered in our algorithm to select an optimal set of sensors, i.e., the right-hand side of
(25) must be greater than the threshold probability $P_{t h}$ to avoid scheduling a longer sensing period for feature detection (see line 17 in Algorithm 2).

Algorithm 2. JOINT OPTIMIZATION OF SENSOR SELECTION AND SENSING TIME.

Initialize the desired detection parameters $P_{F A}, P_{M D}, P_{t h}$

2: Initialize the set of available sensors $\Phi=\left\{\chi_{1}, \ldots, \chi_{n_{s}}\right\}$

Initialize the optimal set of sensors $\Omega^{*} \leftarrow \emptyset$

4: Initialize the set of sensing time $\mathbb{T}_{S} \in[1,2,3,4,5] \mathrm{ms}$

Initialize the sensing overhead $\mathcal{O}^{*} \leftarrow \infty$

6: while $\Phi \neq \emptyset$ do

8. $\quad \chi^{*} \leftarrow \arg \max _{\chi_{i} \in \Phi}\left\{P_{i}\right\} / / P_{i}=P_{R} \cdot e^{Y_{i}}$

8: $\quad \Phi \leftarrow \Phi \backslash\left\{\chi^{*}\right\}$

$\Omega \leftarrow \Omega^{*} \cup\left\{\chi^{*}\right\}$

10: $\quad$ for each $T_{S} \in \mathbb{T}_{S}$ do

$$
T_{D}\left(\Omega, T_{S}\right) \leftarrow T_{S}+|\Omega| \times T_{R}
$$

12: $\quad N^{*} \leftarrow \min \left\{\max \left\{\mathbb{E}\left[N\left(\Omega, P_{F A}, P_{M D}, T_{S}\right)\right], 1\right\}, N_{\max }\right\}$

$\mathcal{O}_{E}\left(\Omega, T_{S}\right) \leftarrow N^{*} \times T_{D}\left(\Omega, T_{S}\right)$

14: end for

$T_{S}^{*} \leftarrow \arg \min _{T_{S} \in \mathbb{T}_{S}}\left\{\mathcal{O}_{E}\left(\Omega, T_{S}\right)\right\}$

16: $\quad \mathcal{O}_{\min } \leftarrow \mathcal{O}_{E}\left(\Omega, T_{S}^{*}\right)$

if $\mathcal{O}_{\text {min }}>\mathcal{O}^{*}$ and $\operatorname{Pr}\left(N_{\text {opt }} \leq N_{\max }\right) \geq P_{\text {th }}$ then

18: $\quad$ return $\left(\Omega^{*}, T_{S}^{*}\right)$

else

20: $\quad \Omega^{*} \leftarrow \Omega$

$\mathcal{O}^{*} \leftarrow \mathcal{O}_{\min }$

22: $\quad$ end if

end while

\subsubsection{Finding the Optimal Threshold $P_{t h}^{*}$}

Without reaching one of the thresholds, $\Gamma_{u}$ and $\Gamma_{l}$, the fusion center must schedule a fine-sensing period. Thus, for a given threshold value of $P_{t h}$, the total expected sensing overhead to meet the detection requirements can be expressed as:

$$
\mathcal{O}_{\text {total }}=\mathcal{O}_{E}\left(P_{t h}\right)+\left(1-P_{t h}\right) \cdot \mathcal{O}_{F},
$$

where $\mathcal{O}_{E}\left(P_{t h}\right)$ is the sensing overhead of energy detection with cooperative sensing and sensing scheduling, subject to the constraint $P_{t h}$; a higher $P_{t h}$ incurs a larger overhead. On the other hand, $\mathcal{O}_{F}$ is the sensing overhead of feature detection, which is assumed to be a constant, e.g., feature detection takes up to $26 \mathrm{~ms}$ for DTV signal detection.

The first and second terms in (26) are monotonically increasing and decreasing functions of $P_{t h}$. Therefore, there exists an optimal $P_{t h}^{*}$ that minimizes the total sensing overhead. We will study the impact of $P_{t h}$ on the sensing overhead in Section 6.

\subsection{Algorithm for Joint Optimization of Sensor Selection and Sensing Time}

We now turn to the problem of finding an optimal set of sensors, as well as an optimal sensing time, that together minimize the average sensing overhead. Let $\Phi$ denote the total set of sensors in the network available for cooperative sensing with estimated RSS distributions via training. The key idea is to utilize a subset $\Omega \subseteq \Phi$ of sensors with relatively high-average RSS values, and also select the sensing time $T_{S}$, thus minimizing both the number of 
cooperating sensors and the number of sensing periods in incumbent detection, while guaranteeing the detectability requirements. Given a subset of sensors, $\Omega$, and sensing time $T_{S}$, the total expected sensing overhead before the fusion center accumulates enough sensing samples can be expressed as:

$$
\mathcal{O}_{E}\left(\Omega, T_{S}\right)=\min \left\{\max \{\mathbb{E}[N(\Omega)], 1\}, N_{\max }\right\} \times T_{D}\left(\Omega, T_{S}\right),
$$

where $\mathbb{E}[N(\Omega)]$ is the expected number of sensing periods necessary to meet the detection requirement with a set $\Omega$ of sensors. $T_{D}\left(\Omega, T_{S}\right)$ is the total time duration for a single sensing, which consists of a sensing period and a measurement reporting period:

$$
T_{D}\left(\Omega, T_{S}\right)=T_{S}+|\Omega| \times T_{R},
$$

where $T_{S}$ is the sensing duration and $T_{R}$ is the duration of a time slot for reporting the sensing result to the fusion center.

Then, based on (25), (27), and (28), our problem of finding an optimal set of sensors and optimal sensing time can be formally stated as:

$$
\begin{array}{cl}
\text { Find } & \left(\Omega^{*}, T_{S}^{*}\right)=\arg \min _{\Omega \subseteq \Phi, T_{S} \in \mathbb{T}_{S}} \mathcal{O}_{E}\left(\Omega, T_{S}\right) \\
\text { subjectto } & \operatorname{Prob}\left(N_{\text {opt }} \leq N_{\text {max }}\right) \geq P_{\text {th }}, \\
& P_{F A} \leq \alpha, \\
& P_{M D} \leq \beta,
\end{array}
$$

where $\alpha$ and $\beta$ are the desired false-alarm and misdetection probabilities.

Unfortunately, there is no analytical solution to this problem, and finding an optimal solution is computationally prohibitive as it requires an exhaustive combinatorial search. For this, we propose a simple algorithm as described in Algorithm 2. The idea is that we sort the sensors in descending order of average RSS and then add sensors to $\Omega$ from the top of the list until the total sensing overhead increases by adding another sensor, and the detection constraint (i.e., $\left.P_{t h}\right)$ is satisfied (line 17). The algorithm provides an optimal solution with a low computational overhead, i.e., $O\left(|\Phi| \cdot\left|\mathbb{T}_{S}\right|\right)$, where $|\Phi|$ and $\left|\mathbb{T}_{S}\right|$ are the total number of available sensors and sensing times, respectively. On the other hand, the exhaustive search requires $O\left(2^{|\Phi| \cdot\left|\mathbb{T}_{S}\right|}-1\right)$. The algorithm is shown to reduce the sensing overhead significantly (see Section 6.3), while guaranteeing the desired level of detection performance.

\section{Performance Evaluation}

This section comparatively evaluates the proposed algorithms using MATLAB-based simulation under realistic wireless environments.

\subsection{Simulation Setup}

We consider a DSA network with a large-scale primary transmitter (e.g., a TV transmitter) and multiple SUs (or sensors). To demonstrate the efficacy of the proposed schemes in realistic wireless environments, we consider the network parameters, which are used widely in IEEE 802.22 WRANs. We assume that the noise power is $N B_{i}=$ $-95.2 \pm \Delta_{i} \mathrm{dBm}$, which is commonly used in IEEE 802.22 WRANs [14], where $\Delta_{i}$ is the noise uncertainty (in $\mathrm{dB}$ ) at sensor $i$ and $B$ is the channel bandwidth. The channel

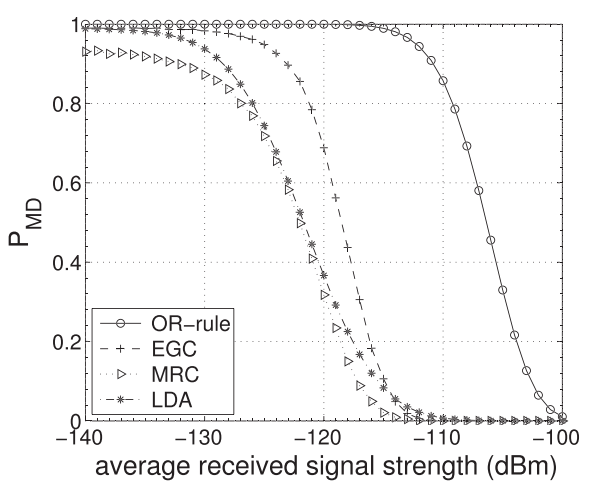

Fig. 10. Performance of detection schemes for one-time sensing with 10 sensors. RSS-profile-based cooperative sensing (denoted as LDA) shows near-optimal performance even in a very low SNR environment.

bandwidth is set to $B=6 \mathrm{MHz}$ as in TV channels. We consider shadow fading with dB-spread $\sigma_{d B}=5.5(\mathrm{~dB})$, which is also typical in rural area networks such as 802.22 WRANs [46]. Throughout the simulation, we assume that the time slot duration for reporting a RSS measurement $\left(T_{R}\right)$ is fixed at $0.2 \mathrm{~ms}$, and assume $n_{s}=10$ cooperating sensors, unless specified otherwise. For RSS profiling, $10^{4}$ samples for estimating the RSS distributions. This consumes only a total sensing time of 10 seconds, assuming the sensing time of $T_{S}=1 \mathrm{~ms}$. We fix the desired false-alarm probability $P_{F A}=0.01$ throughout the evaluation.

To demonstrate the benefits of the proposed sensing algorithms, we evaluate the performance of the following decision- and data-fusion rules:

1. OR-rule,

2. Equal Gain Combining (EGC),

3. Maximal Ratio Combining (MRC), and

4. RSS-profile-based sensing.

The OR-rule is a simple decision fusion rule, in which the fusion center concludes that there exists a primary signal if at least one sensor reports the existence of a primary signal. The other two data-fusion rules, EGC and MRC, are different in that EGC does not require any channel state information at the sensors, whereas MRC requires channel state information. EGC and MRC are known to be nearoptimal in high and low SNR regions, respectively [57], serving as the performance benchmark.

\subsection{Performance of RSS-Profile-Based Detection for One-Time Sensing}

We first evaluate the performance of the proposed LDAbased detection scheme for one-time sensing, assuming that the sensors are randomly selected.

\subsubsection{Performance Comparison}

Fig. 10 compares the performance of the testing schemes for different average RSS values. As expected, misdetection probability, $P_{M D}$, increases as average primary signal strength, $P_{R}$, decreases for all the tested schemes. The figure also indicates that the OR-rule ${ }^{8}$ performs worst because it does not fully utilize the sensing results.

8. In the OR-rule, we set the detection threshold to keep the false-alarm probability below 0.01 as in [58]. 


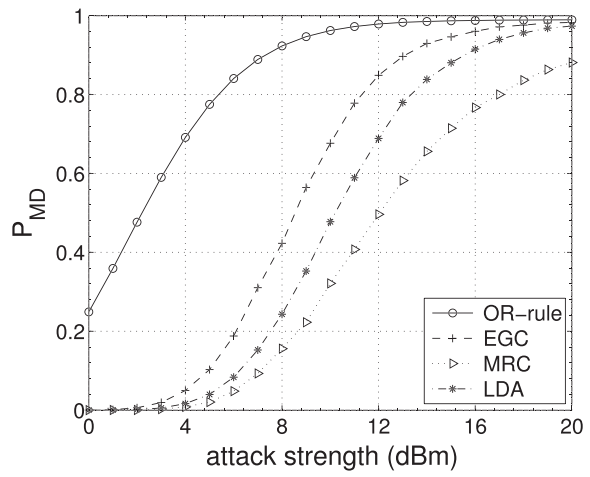

Fig. 11. Effect of attacks on incumbent detection performance. RSSprofile-based spectrum sensing tolerates sensing-targeted attacks well thanks to its ability to exploit spatial diversity in spectrum sensing.

Among the data-fusion rules, MRC outperforms EGC because MRC exploits the SNR information at the sensors, whereas EGC does not require channel estimation. It also shows that our RSS-profile-based detection (denoted as LDA) significantly outperforms the OR-rule and EGC, thanks to its ability to set the near-optimal detection threshold (i.e., an $\left(n_{s}-1\right)$-dimensional hyperplane) based on the spatial RSS profile, which incurs only a minimal training overhead at the time of sensor deployment. More importantly, the performance of the RSS-profile-based scheme is close to MRC, demonstrating its near-optimal detection performance despite its simple detection rule.

In practice, sensors can be compromised by attackers or malfunctioning due to hardware or software defects. Fig. 11 compares the incumbent detection performance (for $P_{F A}$ fixed at 0.01) under different attack scenarios. In the figure, sensing reports are negatively biased by a certain magnitude (in $\mathrm{dBm}$ ) (denoted as attack strength) to emulate collaborative attack scenarios (or erroneous sensors) [29]. The figure shows that the proposed RSS-profile-based spectrum sensing keeps the misdetection probability low for reasonably strong attack strengths (i.e., $<5 \mathrm{~dB}$ ), demonstrating its high robustness. In practice, when the attack strength grows beyond a certain limit, the fusion center can detect such deviations very easily, thus preserving the detection performance.

\subsubsection{Effects of Noise Uncertainty}

Noise uncertainty is one of the main obstacles in using energy detection in a very low SNR environment such as 802.22 WRANs [48]. Noise uncertainty creates a performance barrier called $S N R_{\text {wall }}$, below which signal detection is infeasible irrespective of the sensing time or the number of cooperative sensors. $S N R_{\text {wall }}$, in fact, depends solely on noise uncertainty as [48], specifically, $S N R_{\text {wall }}=\frac{\rho^{2}-1}{\rho}$, where $\rho=10^{\Delta / 10}$ and $\Delta$ (in $\mathrm{dB}$ ) is the noise uncertainty. We assume that the noise uncertainty is bounded by $1 \mathrm{~dB}$ for all sensors, ${ }^{9}$ with the corresponding $S N R_{\text {wall }}$ of $-98.5 \mathrm{dBm}$.

Fig. 12 shows that, when the OR-rule is employed, the detector completely fails to detect the signals below $S N R_{\text {wall }}$

9. This is a reasonable assumption since noise uncertainty can be bounded by $\pm 1(\mathrm{~dB})$, considering several contributing factors such as calibration error, thermal noise variation, changes in LNA amplifier gain, and so on [59].

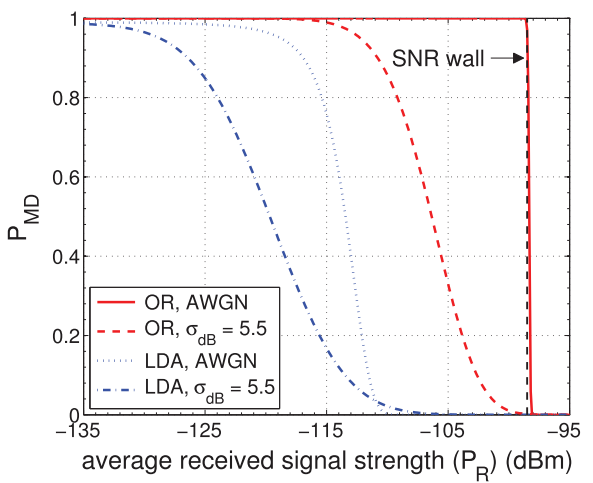

Fig. 12. Effect of noise uncertainty for AWGN and shadow fading environments. RSS-profile-based spectrum sensing works well even when the average RSS is below $S N R_{\text {wall }}$, thus overcoming noise uncertainty.

under the AWGN channel. However, under a practical assumption that noise uncertainty is independent at the sensors, the RSS-profile-based detection works well for signals below $S N R_{\text {wall }}$, even with a one-time sensing, thanks to its ability to exploit spatial RSS diversity. The detection performance improves further under the shadow fading, i.e., $\sigma_{d B}=5.5 \mathrm{~dB}$, as the shadow fading increases the spatial RSS diversity.

\subsubsection{Effects of Sensing Time}

Fig. 13 plots the misdetection performance for various sensing time durations, $T_{S} \in[1,20] \mathrm{ms}$. As we observed in (6), the detection performance depends not only on the average primary signal strength, $P_{R}$, but also on the sensing time. The figure shows that, as the sensing time increases, the misdetection rate decreases for all tested values of $P_{R}$. This is because the more samples the detector is provided with, the more accurate the sensing results, thus eliminating ambiguity regarding the existence of a primary signal. As described in Algorithm 2, the fusion center finds a combination of an optimal set of sensors and an optimal sensing time $T_{S}^{*}$ that minimizes the average sensing overhead.

\subsection{Performance of Online Sensing Scheduling with Optimal Sensor Selection}

We now evaluate the performance of the proposed online sensing scheduling by jointly optimizing the selection of sensors and sensing time.

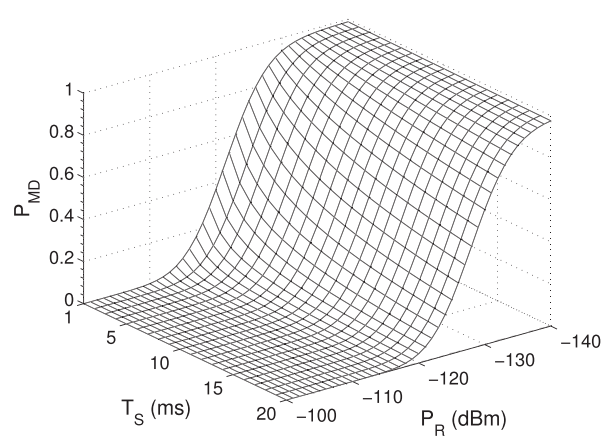

Fig. 13. Impact of sensing time on detection performance. The amount of time a sensor spends on sensing for one-time sensing affects the detection performance. 


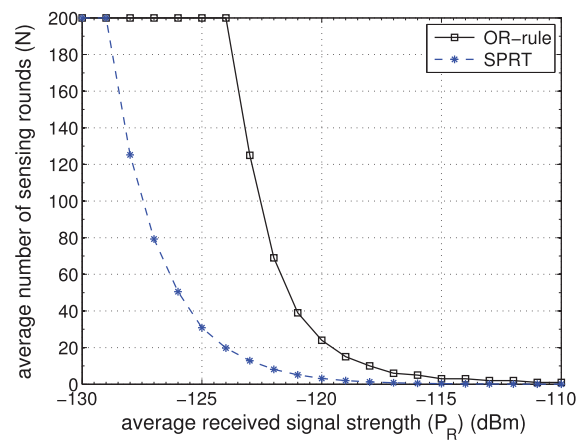

Fig. 14. Average number of sensing periods scheduled to meet the detectability requirement of $P_{M D}, P_{F A} \leq 0.01$. The proposed algorithm significantly reduces the sensing overhead.

\subsubsection{Impact on Incumbent Detection Delay}

Fig. 14 shows the average number of sensing periods that needs to be scheduled to meet the detection requirements. The figure shows that our SPRT-based online sensing scheduling algorithm significantly reduces the average number of sensing periods compared to the OR-rule-based scheduling scheme, thanks to its ability to fully utilize the sensing results via RSS profiles. As a result, our algorithm expands the feasible region of the energy detector significantly. On the other hand, the OR-rule benefits relatively less from scheduling sensing periods because RSSs do not change over time (except the measurement errors) at fixed sensor locations.

\subsubsection{Impact on Sensing Overhead}

We now demonstrate the performance of our optimal sensorselection algorithm in terms of the reduction of sensing overhead. The sensing overhead is defined as the average fraction of time (in percent) spent on fast sensing (i.e., energy detection) within a 2-second interval, which is the channel detection time (CDT) period (see (27)). Here, we set the threshold $P_{t h}=0.95$. Fig. 15 shows the sensing time overhead for various sensing durations $T_{S} \in[1,3,5] \mathrm{ms}$. The figure shows that a larger sensing time, i.e., $T_{S}=5 \mathrm{~ms}$, is favored in relatively low SNR environments (i.e., $P_{R}<-126 \mathrm{dBm}$ ), whereas a smaller sensing time, i.e., $T_{S}=1 \mathrm{~ms}$, is desirable in relatively high SNR environments, in which sensing scheduling may not be needed to achieve the detection requirements. This is because in a low SNR environment, a larger sensing

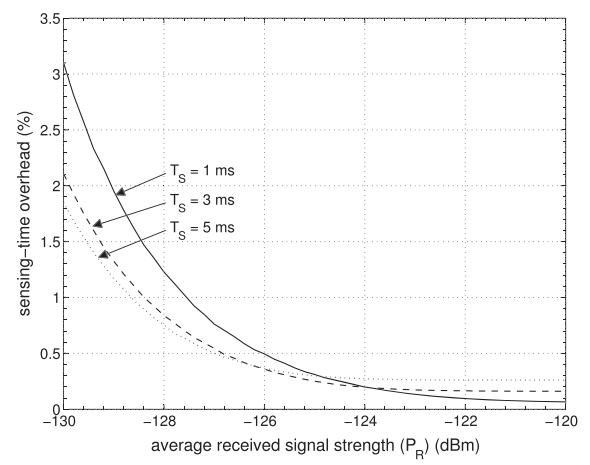

Fig. 15. Impact of sensing time on sensing overhead. A longer sensing time becomes more desirable as the average primary signal strength decreases, and vice versa.

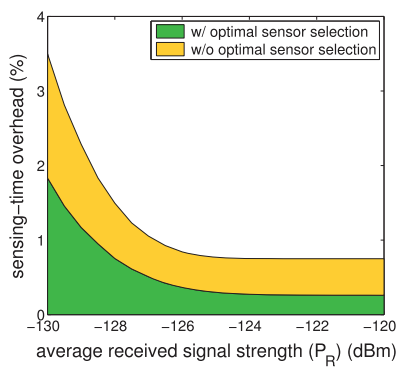

(a) sensing-time overhead

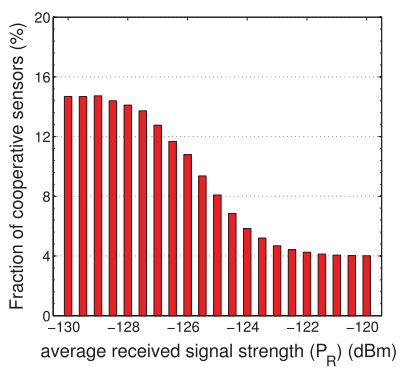

(b) fraction of cooperative sensors
Fig. 16. Performance of the optimal sensor selection algorithm. Our proposed sensor selection algorithm (a) reduces the sensing overhead significantly over the algorithm without sensor selection, and (b) utilizes only a small subset of the sensors (i.e., $<15$ percent). The simulation parameters are set to $P_{t h}=0.95$ and $T_{S}=5 \mathrm{~ms}$.

duration can reduce the number of sensing periods necessary to meet the detection requirement. On the other hand, in a high SNR environment, even one-time sensing might suffice to meet the requirement, so a short sensing duration is more beneficial in minimizing the average overhead.

Fig. 16 compares the average sensing-time overhead, i.e., the fraction of time spent on spectrum sensing and reporting the sensing results within a sensing interval, with the optimal selection of sensors and sensing time against the one without sensor selection. A sensing interval is assumed to be $2 \mathrm{~s}$, which is equivalent to CDT in IEEE 802.22 WRANs. The figure shows that our algorithm minimizes average sensing overhead by up to 94 percent because it selects only a subset of sensors with high average RSSs, thus minimizing both the number of sensing rounds and the sensing result reporting time.

\subsubsection{Impact of $P_{t h}^{*}$ on Sensing Overhead}

We now evaluate the impact of the threshold $P_{t h}$ on the sensing overhead. Fig. 17 plots the sensing-time overhead and the number of cooperative sensors used in our optimal sensor selection algorithm, for the cases in which the total number of sensors in the secondary networks is $n_{s}=20$ and $n_{s}=50$, respectively. Here, we assume that the sensing-time overhead of feature detection is $\mathcal{O}_{F}=20 \mathrm{~ms}$. The sensing overhead is calculated using (26) in Section 5. A small $P_{t h}$ means that the fusion center relies more on feature detection, and vice versa; the fusion center may need to employ more sensors and schedule more sensing periods for energy detection as $P_{t h}$ increases. When $n_{s}=20$, the number of sensors and the total sensing overhead for energy detection, $\mathcal{O}_{E}$, increases with $P_{t h}$. On the other hand, the sensing overhead for feature detection, i.e., $\left(1-P_{t h}\right) \cdot \mathcal{O}_{F}$, decreases linearly with $P_{t h}$, resulting in Fig. 17a.

When $n_{s}=50$, the fusion center can meet the detection requirement with only a small number of sensors selected by the proposed sensor selection algorithm. Thus, the number of sensors is stable over the entire range of $P_{t h}$, as shown in Fig. 17d. Due to the high detection performance of the selected sensors, the number of sensing periods required to meet the detection requirement does not increases with $P_{t h}$. On the other hand, the overhead of feature detection only decreases with $P_{t h}$, resulting in a monotonically decreasing sensing overhead, as shown in Fig. 17c.

In summary, when there is a sufficiently large number of sensors in the secondary network, the fusion center may 


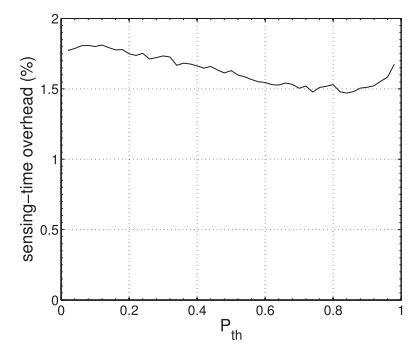

(a) sensing overhead, $n_{s}=20$

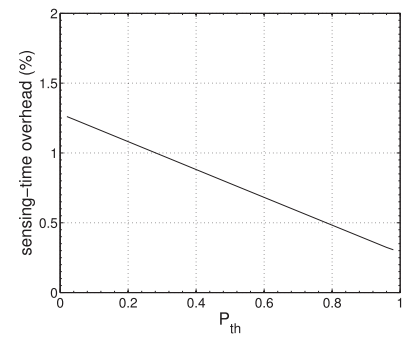

(c) sensing overhead, $n_{s}=50$

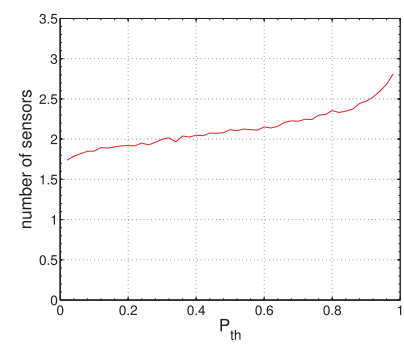

(b) number of sensors, $n_{s}=20$

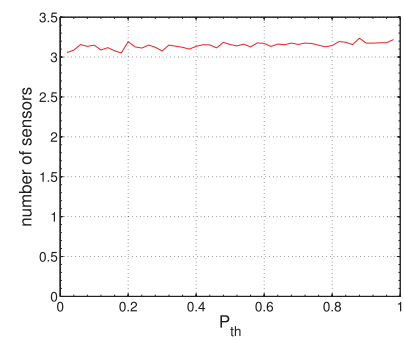

(d) number of sensors, $n_{s}=50$
Fig. 17. Impact of $P_{t h}$ on sensing overhead performance for a given detection requirement. (a)-(b): With a small number of sensors, the fusion center must use both energy and feature detection by adapting $P_{t h}$ based on the network environment. (c)-(d): On the other hand, with a large number of sensors, the fusion center must exploit high-performing sensors for energy detection, to minimize the overall sensing overhead. The simulation parameters are set to $T_{S}=5 \mathrm{~ms}$ and $P_{R}=-130 \mathrm{dBm}$.

prefer to rely on energy detection, and vice versa, to minimize the overall sensing overhead.

\section{Conclusion}

In this paper, we proposed to jointly optimize cooperative sensing and sensing scheduling, to minimize the average sensing overhead, while guaranteeing the desired level of detection requirement. Our spectrum sensing framework exploits the spatiotemporal variations in received primary signal strength by constructing a spatial RSS profile for an incumbent signal. We showed that the RSS distribution of a primary signal can be accurately approximated as a Gaussian distribution in low SNR environments and analyzed the detection performance of the RSS-profilebased detection scheme. We have also shown that there is virtually no correlation between sensing reports from stationary sensors, regardless of their physical locations. Based on these observations, we formulated the problem of sensing scheduling as a sequential hypothesis test, which finds an optimal time to stop scheduling sensing subject to given detection requirements. We also proposed an optimal algorithm that minimizes the average sensing overhead via optimal selection of sensors and sensing duration, by balancing the tradeoff between detection performance and sensing overhead. Our evaluation results have shown that the proposed sensing algorithms reduce sensing overhead by up to 65 percent in practical scenarios.

\section{REFERENCES}

[1] T.M. Taher, R.B. Bacchus, K.J. Zdunek, and D.A. Roberson, “LongTerm Spectral Occupancy Findings in Chicago," Proc. IEEE Symp. New Frontiers in Dynamic Spectrum Access Networks (DySPAN), May 2011.
[2] T. Harrold, R. Cepeda, and M. Beach, "Long-Term Measurement of Spectrum Occupancy Characteristics," Proc. IEEE Symp. New Frontiers in Dynamic Spectrum Access Networks (DySPAN), May 2011.

[3] A. Ghasemi and E.S. Sousa, "Opportunistic Spectrum Access in Fading Channels through Collaborative Sensing," J. Comm., vol. 2, no. 2, pp. 71-82, Mar. 2007.

[4] H. Kim and K.G. Shin, "In-Band Spectrum Sensing in Cognitive Radio Networks: Energy Detection or Feature Detection?" Proc. ACM MobiCom, Sept. 2008.

[5] G.J. Buchwald, S.L. Kuffner, L.M. Ecklund, M. Brown, J. Edgar, and H. Callaway, "The Design and Operation of the IEEE 802.22.1 Disabling Beacon for the Protection of TV Whitespace Incumbents," Proc. IEEE Symp. New Frontiers in Dynamic Spectrum Access Networks (DySPAN), Oct. 2008.

[6] Z. Lei and F. Chin, "A Reliable and Power Efficient Beacon Structure for Cognitive Radio Systems," Proc. IEEE Int'l Conf. Comm. (ICC), May 2008.

[7] R. Murty, R. Chandra, T. Moscibroda, and P. Bahl, "SenseLess: A Database-Driven White Spaces Networks," Proc. IEEE Symp. New Frontiers in Dynamic Spectrum Access Networks (DySPAN), May 2011.

[8] W. Caldwell, "Geolocation with Database Requirement Summary," IEEE 802.22-06/0140r0, July 2006.

[9] D. Gurney, G. Buchwald, L. Ecklund, S. Kuffner, and J. Grosspietsch, "Geo-Location Database Techniques for Incumbent Protection in the TV White Space," Proc. IEEE Symp. New Frontiers in Dynamic Spectrum Access Networks (DySPAN), Oct. 2008.

[10] IEEE 802.22 Working Group on Wireless Regional Area Networks, http://www.ieee802.org/22, 2013.

[11] C.R. Stevenson, G. Chouinard, Z. Lei, W. Hu, S.J. Shellhammer, and W. Caldwell, "IEEE 802.22: The First Cognitive Radio Wireless Regional Area Network Standard," IEEE Comm. Mag., vol. 47 , no. 1, pp. 130-138, Jan. 2009.

[12] C. Cordeiro, K. Challapali, D. Birru, and S. Shankar, "IEEE 802.22: An Introduction to the First Wireless Standard Based on Cognitive Radio," J. Comm., vol. 1, no. 1, pp. 38-47, Apr. 2006.

[13] S. Shellhammer, S. Shankar, R. Tandra, and J. Tomcik, "Performance of Power Detector Sensors of DTV Signals in IEEE 802.22 WRANs," Proc. ACM First Int'l Workshop Technology and Policy for Accessing Spectrum (TAPAS), Aug. 2006.

[14] S. Shellhammer and R. Tandra, "Performance of the Power Detector with Noise Uncertainty," IEEE 802.22-06/0134r0, July 2006.

[15] S. Shellhammer and R. Tandra, "An Evaluation of DTV Pilot Power Detection," IEEE 802.22-06/0188r0, Sept. 2006.

[16] R. Murty, R. Chandra, T. Moscibroda, and P. Bahl, "SenseLess: A Database-Driven White Spaces Networks," Technical Report MSR-TR-2010-127, MSR, Sept. 2010.

[17] Federal Communications Commission, "Second Memorandum Opinion and Order," FCC 10-174, Sept. 2010.

[18] D. Cabric, S.M. Mishra, and R.W. Brodersen, "Implementation Issues in Spectrum Sensing for Cognitive Radios," Proc. Conf. Record 38th Asilomar Conf. Signals, Systems and Computer, Nov. 2004.

[19] A. Ghasemi and E.S. Sousa, "Collaborative Spectrum Sensing for Opportunistic Access in Fading Environments," Proc. IEEE Symp. New Frontiers in Dynamic Spectrum Access Networks (DySPAN), Nov. 2005.

[20] E. Visotsky, S. Kuffner, and R. Peterson, "On Collaborative Detection of TV Transmissions in Support of Dynamic Spectrum Sharing," Proc. IEEE Symp. New Frontiers in Dynamic Spectrum Access Networks (DySPAN), Nov. 2005.

[21] S.M. Mishra, A. Sahai, and R.W. Brodersen, "Cooperative Sensing among Cognitive Radios," Proc. IEEE Int'l Conf. Comm. (ICC), June 2006.

[22] J. Unnikrishnan and V.V. Veeravalli, "Cooperative Sensing for Primary Detection in Cognitive Radio," IEEE J. Selected Topics Signal Processing, vol. 2, no. 1, pp. 18-27, Feb. 2008.

[23] G. Chouinard, "Use of Collaborative Sensing to Reduce False Positive Results," IEEE 802.22-08/0118r0, May 2008.

[24] W. Saad, Z. Han, M. Debbah, A. Hjørungnes, and T. Başar, "Coalition Games for Distributed Collaborative Spectrum Sensing in Cognitive Radio Networks," Proc. IEEE INFOCOM, Apr. 2009.

[25] C. Cordeiro, K. Challapali, and M. Ghosh, "Cognitive PHY and MAC Layers for Dynamic Spectrum Access and Sharing of TV Bands," Proc. ACM First Int'l Workshop Technology and Policy for Accessing Spectrum (TAPAS), Aug. 2006. 
[26] J. Nieminen, R. Jäntti, and L. Qian, "Primary User Detection in Distributed Cognitive Radio Networks Under Timing Inaccuracy," Proc. IEEE Symp. New Frontiers in Dynamic Spectrum Access Networks (DySPAN), Apr. 2010.

[27] Y. Selén, H. Tullberg, and J. Kronander, "Sensor Selection for Cooperative Spectrum Sensing," Proc. IEEE Symp. New Frontiers in Dynamic Spectrum Access Networks (DySPAN), Oct. 2008.

[28] A.W. Min, K.G. Shin, and X. Hu, "Attack-Tolerant Distributed Sensing for Dynamic Spectrum Access Networks," Proc. IEEE 17th Int'l Conf. Network Protocols (ICNP), Oct. 2009.

[29] A.W. Min, K.-H. Kim, and K.G. Shin, "Robust Cooperative Sensing via State Estimation in Cognitive Radio Networks," Proc. IEEE Symp. New Frontiers in Dynamic Spectrum Access Networks (DySPAN), May 2011.

[30] A.W. Min, K.G. Shin, and X. Hu, "Secure Cooperative Sensing in IEEE 802.22 WRANs Using Shadow Fading Correlation," IEEE Trans. Mobile Computing, vol. 10, no. 10, pp. 1434-1447, Oct. 2011.

[31] Y.-C. Liang, Y. Zeng, E.C. Peh, and A.T. Hoang, "SensingThroughput Tradeoff for Cognitive Radio Networks," IEEE Trans. Wireless Comm., vol. 7, no. 4, pp. 1326-1337, Apr. 2008.

[32] S. Huang, X. Liu, and Z. Ding, "Optimal Transmission Strategies for Dynamic Spectrum Access in Cognitive Radio Networks," IEEE Trans. Mobile Computing, vol. 8, no. 12, pp. 1636-1648, Dec. 2009.

[33] A.W. Min and K.G. Shin, "On Sensing-Access Tradeoff in Cognitive Radio Networks," Proc. IEEE Symp. New Frontiers in Dynamic Spectrum Access Networks (DySPAN), Apr. 2010.

[34] A. Ghasemi and E.S. Sousa, "Asymptotic Performance of Collaborative Spectrum Sensing under Correlated Log-Normal Shadowing," IEEE Comm. Letters, vol. 11, no. 1, pp. 1089-7798, Jan. 2007.

[35] I.F. Akyildiz, B.F. Lo, and R. Balakrishnan, "Cooperative Spectrum Sensing in Cognitive Radio Networks: A Survey," Elsevier Physical Comm., vol. 4, no. 1, pp. 40-62, Mar. 2011.

[36] A.T. Hoang and Y.-C. Liang, "Adaptive Scheduling of Spectrum Sensing Periods in Cognitive Radio Networks," Proc. IEEE Globecom, Nov. 2007.

[37] L. Lai, Y. Fan, and H.V. Poor, "Quickest Detection in Cognitive Radio: A Sequential Change Detection Framework," Proc. IEEE Globecom, Nov. 2008.

[38] Y. Xin, H. Zhang, and S. Rangarajan, "SSCT: A Simple Sequential Spectrum Sensing Scheme for Cognitive Radio," Proc. IEEE GlobeCom, Nov. 2009.

[39] K.W. Choi, W.S. Jeon, and D.G. Jeong, "Sequential Detection of Cyclostationary Signal for Cognitive Radio Systems," IEEE Trans. Wireless Comm., vol. 8, no. 9, pp. 4480-4485, Sept. 2009.

[40] Q. Zou, S. Zheng, and A.H. Sayed, "Cooperative Sensing via Sequential Detection," IEEE Trans. Signal Processing, vol. 58, no. 12, pp. 6266-6283, Dec. 2010.

[41] J. Benko et al., "Draft PHY/MAC Specification for IEEE 802.22," IEEE 802.22-06/0069r1, May 2006.

[42] K.B. Letaief and W. Zhang, "Cooperative Communications for Cognitive Radio Networks," Proc. IEEE, vol. 97, no. 5, pp. 878-893, May 2009.

[43] J. Lundén, V. Koivunen, A. Huttunen, and H.V. Poor, "Collaborative Cyclostationary Spectrum Sensing for Cognitive Radio Systems," IEEE Trans. Signal Processing, vol. 57, no. 11, pp. 41824195, Nov. 2009.

[44] F. Zeng, C. Li, and Z. Tian, "Distributed Compressed Spectrum Sensing in Cooperative Multihop Cognitive Networks," IEEE J. Selected Topics Signaling, vol. 5, no. 1, pp. 37-487, Feb. 2011.

[45] S. Shellhammer, "Performance of the Power Detector," IEEE 802.22-06/0075r0, May 2006.

[46] S. Shellhammer, V. Tawil, G. Chouinard, M. Muterspaugh, and M. Ghosh, "Spectrum Sensing Simulation Model," IEEE 802.22-06/ 0028r10, Sept. 2006.

[47] F.F. Digham, M.-S. Alouini, and M.K. Simon, "On the Energy Detection of Unknown Signals over Fading Channels," IEEE Trans. Comm., vol. 55, no. 1, pp. 21-24, Jan. 2007.

[48] R. Tandra and A. Sahai, "SNR Walls for Signal Detection," IEEE J. Selected Areas Comm., vol. 2, no. 1, pp. 4-17, Feb. 2008.

[49] T. Hastie, R. Tibshirani, and J. Friedman, The Elements of Statistical Learning. Springer, 2001.

[50] M.M.E. Ayadi, M.S. Kamel, and F. Karray, "Toward a Tight Upper Bound for the Error Probability of the Binary Gaussian Classification Problem," Pattern Recognition, vol. 41, no. 6, pp. 2120-2132, June 2008 .
[51] M. Gudmundson, "Correlation Model for Shadow Fading in Mobile Radio Systems," Electronic Letters, vol. 27, no. 23, pp. 21452146, Nov. 1991.

[52] A. Safak, "Statistical Analysis of the Power Sum of Multiple Correlated Log-Normal Components," IEEE Trans. Vehicular Technology, vol. 42, no. 1, pp. 58-61, Feb. 1993.

[53] L.F. Fenton, "The Sum of Log-Normal Probability Distributions in Scatter Transmission Systems," IRE Trans. Comm. Systems, vol. 8, no. 1, pp. 57-67, Mar. 1960.

[54] GNU Software Radio Project, http://www.gnu.org/software/ gnuradio/, 2013.

[55] A. Wald, Sequential Analysis. Dover, 2004.

[56] S. Shellhammer and R. Tandra, "Numerical Spectrum Sensing Requirements," IEEE 802.22-06/0088r0, June 2006.

[57] J. Ma, G. Zhao, and Y.G. Li, "Soft Combination and Detection for Cooperative Spectrum Sensing in Cognitive Radio Networks," IEEE Trans. Wireless Comm., vol. 7, no. 11, pp. 4502-4507, Nov. 2008.

[58] W. Zhang, R.K. Mallik, and K.B. Letaief, "Cooperative Spectrum Sensing Optimization in Cognitive Radio Networks," Proc. IEEE Int'l Conf. Comm. (ICC), May 2008.

[59] S. Shellhammer and G. Chouinard, "Spectrum Sensing Requirements Summary," IEEE 802.22-06/0089r5, July 2006.

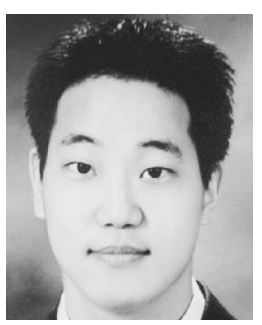

Alexander W. Min received the BS degree in electrical engineering from Seoul National University, Korea, in 2005, and the PhD degree in electrical engineering and computer science from the University of Michigan, Ann Arbor, in 2011. He is currently a research scientist in the Systems Architecture Lab at Intel Labs. In 2010, he was a research intern at Deutsche Telekom, Inc., R\&D Labs, Los Altos, California. His research interests include cognitive radio and dynamic spectrum access networks, wireless security, low-power mobile platforms, and mobile sensing. He has served as a reviewer for leading networking journals and conferences and on the technical program committees for conferences in wireless communications and mobile computing. He is a member of the IEEE, ACM, and the IEEE Communications Society.

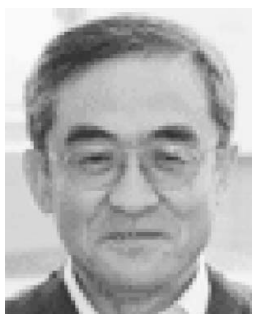

Kang G. Shin is the Kevin \& Nancy O'Connor professor of computer science in the Department of Electrical Engineering and Computer Science, The University of Michigan, Ann Arbor. His current research focuses on computing systems and networks as well as on embedded real-time and cyberphysical systems, all with emphasis on timeliness, security, and dependability. He has supervised the completion of $71 \mathrm{PhDs}$, and authored/coauthored more than 770 technical articles (more than 270 of these are in archival journals), one textbook, and more than 20 patents or invention disclosures, and received numerous awards, including Best Paper Awards from the 2011 ACM International Conference on Mobile Computing and Networking (MobiCom '11), the 2011 IEEE International Conference on Autonomic Computing, the 2010 and 2000 USENIX Annual Technical Conferences, as well as the 2003 IEEE Communications Society William R. Bennett Prize Paper Award and the 1987 Outstanding IEEE Transactions of Automatic Control Paper Award. He has also received several institutional awards, including the Research Excellence Award in 1989, the Outstanding Achievement Award in 1999, the Distinguished Faculty Achievement Award in 2001, and the Stephen Attwood Award in 2004 from The University of Michigan (the highest honor bestowed to Michigan Engineering faculty); a Distinguished Alumni Award of the College of Engineering, Seoul National University in 2002; the 2003 IEEE RTC Technical Achievement Award; and the 2006 Ho-Am Prize in Engineering (the highest honor bestowed to Korean-origin engineers). $\mathrm{He}$ has chaired several major conferences, including 2009 ACM MobiCom, 2008 IEEE SECON, 2005 ACM/USENIX MobiSys, 2000 IEEE RTAS, and 1987 IEEE RTSS. He has served on editorial boards, including the IEEE Transactions on Parallel and Distributed Systems and ACM Transactions on Embedded Systems. He has also served on numerous government committees, such as the US National Science Foundation Cyber-Physical Systems Executive Committee and the Korean Government R\&D Strategy Advisory Committee. He has also cofounded a couple of startups. He is a fellow of the IEEE and the ACM. 